\title{
Cdc42 regulates bone modeling and remodeling in mice by modulating RANKL/ M-CSF signaling and osteoclast polarization
}

\author{
Yuji Ito,, ${ }^{1}$ Steven L. Teitelbaum, ${ }^{1}$ Wei Zou, ${ }^{1}$ Yi Zheng, ${ }^{2}$ James F. Johnson, ${ }^{2}$ \\ Jean Chappel, ${ }^{1}$ F. Patrick Ross, ${ }^{1}$ and Haibo Zhao' \\ 1Department of Pathology and Immunology, Washington University School of Medicine, St. Louis, Missouri, USA. \\ 2Division of Experimental Hematology, Children's Hospital Medical Center, \\ Molecular Developmental Biology Graduate Program, University of Cincinnati, Cincinnati, Ohio, USA.
}

\begin{abstract}
The modeling and remodeling of bone requires activation and polarization of osteoclasts, achieved by reorganization of the cytoskeleton. Members of the Rho subfamily of small GTPases, including Cdc42, are known regulators of cytoskeletal components, but the role of these proteins in bone physiology and pathophysiology remains unclear. Here, we examined loss-of-function mice in which $\mathrm{Cdc} 42$ was selectively ablated in differentiated osteoclasts and gain-of-function animals wherein Cdc42Gap, a protein that inactivates the small GTPase, was deleted globally. Cdc42 loss-of-function mice were osteopetrotic and resistant to ovariectomy-induced bone loss, while gain-of-function animals were osteoporotic. Isolated Cdc42-deficient osteoclasts displayed suppressed bone resorption, while osteoclasts with increased $\mathrm{Cdc} 42$ activity had enhanced resorptive capacity. We further demonstrated that Cdc42 modulated M-CSF-stimulated cyclin D expression and phosphorylation of Rb and induced caspase 3 and Bim, thus contributing to osteoclast proliferation and apoptosis rates. Furthermore, Cdc42 was required for multiple $\mathrm{M}-\mathrm{CSF}$ - and RANKL-induced osteoclastogenic signals including activation and expression of the differentiation factors MITF and NFATc1 and was a component of the Par3/Par6/atypical PKC polarization complex in osteoclasts. These data suggest that $\mathrm{Cdc42}$ regulates osteoclast formation and function and may represent a promising therapeutic target for prevention of pathological bone loss.
\end{abstract}

\section{Introduction}

Generation and maintenance of skeletal mass and integrity requires continuous renewal of bone by a combination of modeling and remodeling that is mediated by osteoblasts and osteoclasts. In osteoporosis, resorption exceeds osteogenesis, while defective osteoclast formation and/or function results in bone accumulation (1)

Osteoclasts are multinucleated cells generated from mononuclear precursors of the monocyte/macrophage lineage. RANKL and M-CSF, the two principal osteoclastogenic cytokines, trigger signals that control precursor differentiation and activation of the mature resorptive cell $(2,3)$. Activated osteoclasts polarize by reorganizing their cytoskeleton. Upon matrix recognition, actin polymerizes into podosomal rings, adhesion structures at the sealing zone where the plasma membrane juxtaposes bone. This sealing zone surrounds a specialized domain, the ruffled border, through which protons and lysosomal enzymes are secreted into the resorption lacuna to dissolve bone mineral and digest organic matrix, respectively (4).

Rho, Rac, and Cdc42, the most studied Rho subfamily small GTPases, are key regulators of the actin cytoskeleton. Acting as molecular switches, by cycling between active, GTP-bound and inactive, GDP-bound forms, they play multiple roles in cell regulation (5). These inter-conversions are controlled by guanine nucleotide-exchange factors (GEFs) and GTPase-activating proteins (GAPs) (6). While the role of Rho, Rac, and Cdc42 in actin organization has been examined in a range of cell types, studies

Conflict of interest: The authors have declared that no conflict of interest exists. Citation for this article: J Clin Invest. 2010;120(6):1981-1993. doi:10.1172/JCI39650. in osteoclasts have invariably involved in vitro experiments using chemical inhibitors or dominant-negative and/or overexpression of constitutively active mutants of the small GTPases $(7,8)$. Unfortunately, these approaches have yielded contradictory conclusions. Thus, two groups have suggested that Rho-A is critical for osteoclast podosome organization, motility, and bone resorption $(9,10)$. In contrast, Destaing et al. proposed that inhibited Rho-A yields podosome patterning via Rho-mDia2-HDAC6 microtubule acetylation (11). Similarly, ligation of the integrin $\alpha v \beta 3$ activates Rac, which is required for actin ring formation (12), whereas dominantnegative Rac does not affect the osteoclast cytoskeleton, but rather regulates osteoclast apoptosis (8). The Wiscott-Aldrich syndrome protein (WASP) and actin-related protein 2/3 (Arp2/3) complex participate in actin ring formation and bone resorption $(13,14)$. Because they are Cdc42 effectors, this GTPase may regulate the resorptive polykaryon.

Here we used mice in which Cdc42 is absent only in mature osteoclasts (loss of function) or present as a basally active molecule (gain of function) in committed precursors to characterize the role of the GTPase, in vivo and in vitro. We find that the molecule, which is activated independently by both M-CSF and RANKL, regulates properties of the resorptive cell. Specifically, Cdc42 is essential for skeletal homeostasis during modeling and remodeling, and its deletion provides protection from oophorectomy-induced bone loss. Cdc42 exerts its skeletal effects by regulating proliferation of osteoclast precursors via GSK-3 $\beta /$ cyclin $\mathrm{D} / \mathrm{Rb}$ and apoptosis of mature cells through $\mathrm{Bim} /$ caspase- 9 . It is also a component of the Par-3/Par-6/atypical PKC (Par-3/Par-6/aPKC) polarization complex, which governs the rate of actin ring formation. Cdc42 is therefore an essential component of RANKL and M-CSF signal- 


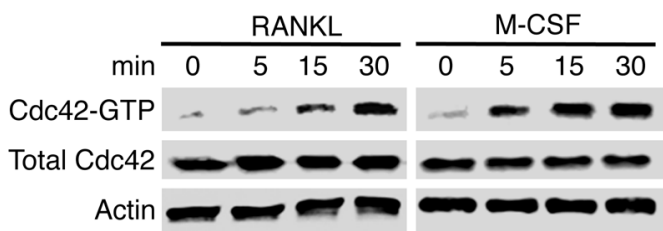

ing, regulating osteoclast recruitment physiologically and in the context of postmenopausal osteoporosis.

\section{Results}

Generation of osteoclast-specific $\mathrm{Cdc} 42^{-/-}$mice and $\mathrm{Cdc} 42 \mathrm{Gap}^{-/-}$radiation chimeras. M-CSF and RANKL are the 2 major regulators of osteoclast differentiation. Kim et al. have shown the latter acti-

\section{Figure 1}

Cdc42 is activated by RANKL or M-CSF. Cytokine- and serum-starved WT pre-osteoclasts were exposed to RANKL $(100 \mathrm{ng} / \mathrm{ml})$ or M-CSF ( $50 \mathrm{ng} / \mathrm{ml}$ ) over time. Cdc42-GTP was assessed by pulldown assay. Total Cdc42 and $\beta$-actin served as loading controls.

vates Cdc42 (15), and we found the same is true regarding M-CSF. As seen in Figure 1, within 5-15 minutes each cytokine activated the small GTPase.

These experiments suggest $\mathrm{Cdc} 42$ may regulate the osteoclast. To determine whether this is the case, we generated osteoclasts in which activity of the GTPase is suppressed or enhanced. Since conventional gene targeting of $\mathrm{Cdc} 42$ prompts early embryonic lethality,
A
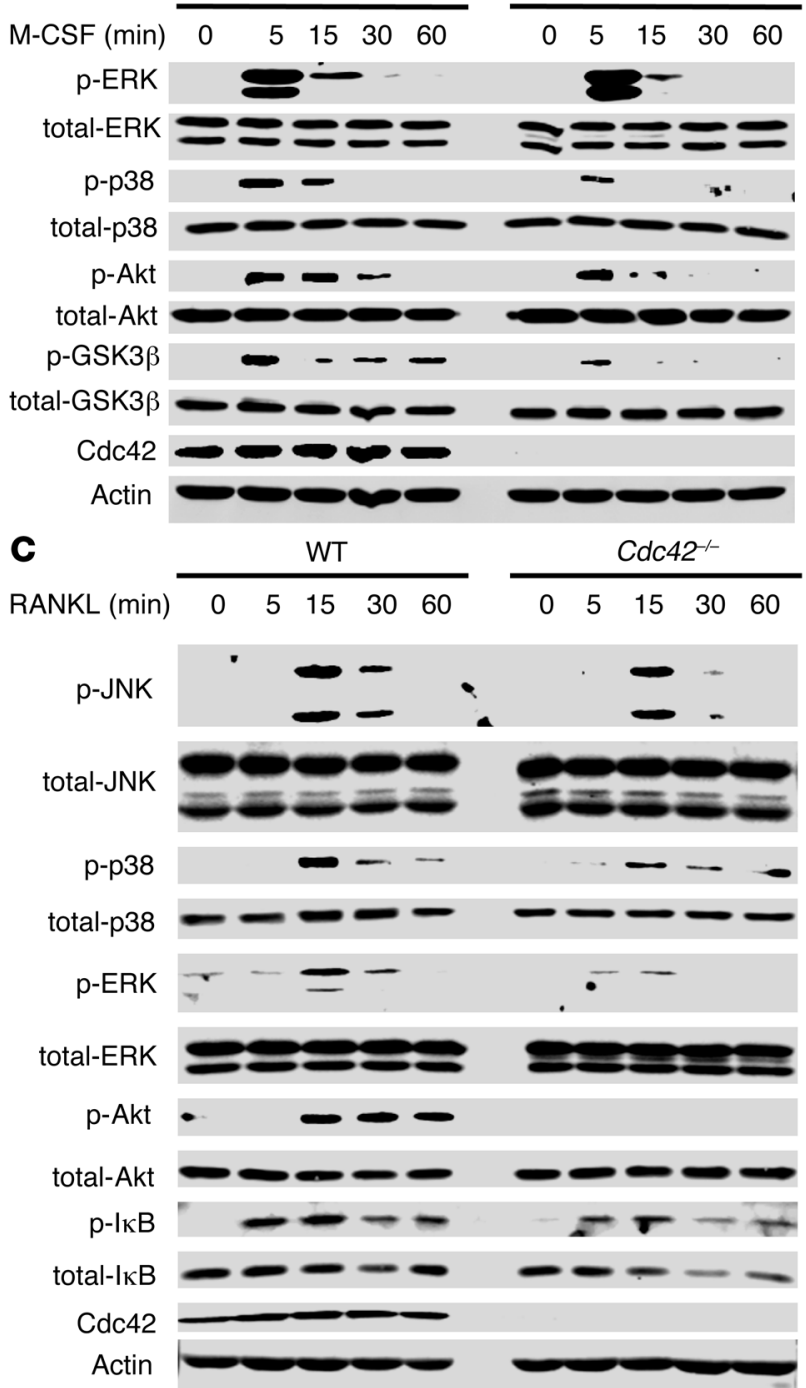

B

M-CSF (min)

p-ERK

total-ERK

p-p38

total-p38

p-Akt

total-Akt

p-GSK3 3

total-GSK3 $\beta$

Actin

\begin{tabular}{lllll} 
WT \\
\hline 0 & 5 & 15 & 30 & 60
\end{tabular}
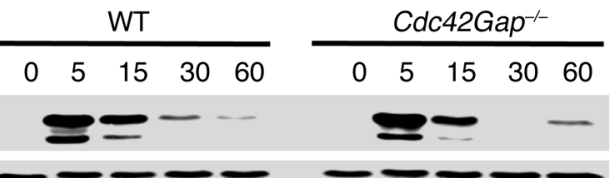

E-

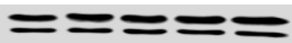

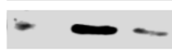

D

RANKL (min)

\begin{tabular}{lllll} 
WT & \\
\hline 0 & 5 & 15 & 30 & 60
\end{tabular}

$\infty$

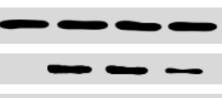

p-JNK

500

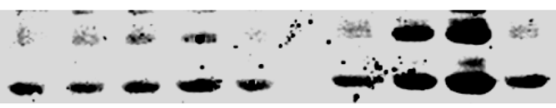

total-JNK
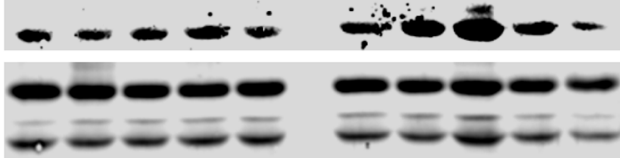

p-p38

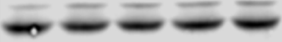
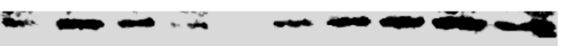

total-p38

p-ERK

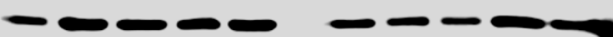

total-ERK
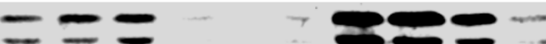

total-ERK
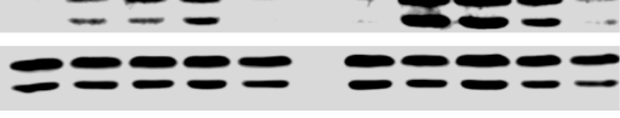

p-Akt
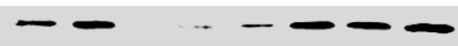

total-Akt

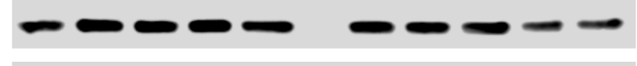

$\mathrm{p}-\mathrm{I} \kappa \mathrm{B}$

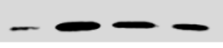

$-$

total-IкB

Actin

Figure 2

Cdc42 modulates multiple RANKL and M-CSF signaling pathways. (A and B) Cytokine- and serum-starved BMMs were exposed to M-CSF $(50 \mathrm{ng} / \mathrm{ml})$ over time. Total and phosphorylated signaling molecules were detected by Western blots. $\beta$-Actin served as loading control. (C and D) Cytokine- and serum-starved BMMs were exposed to RANKL (100 ng/ml) over time. Total and phosphorylated signaling molecules were detected by Western blots. $\beta$-Actin served as loading control. 

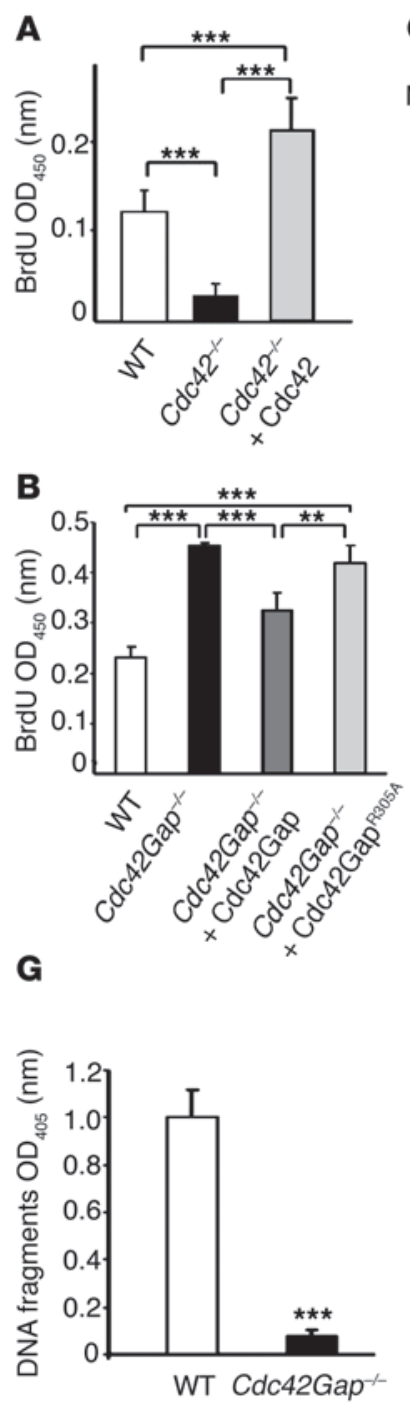

C

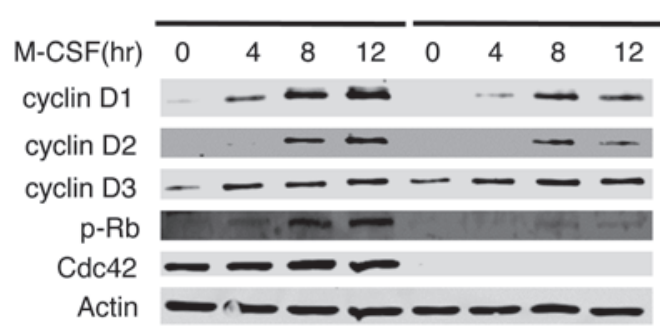

D

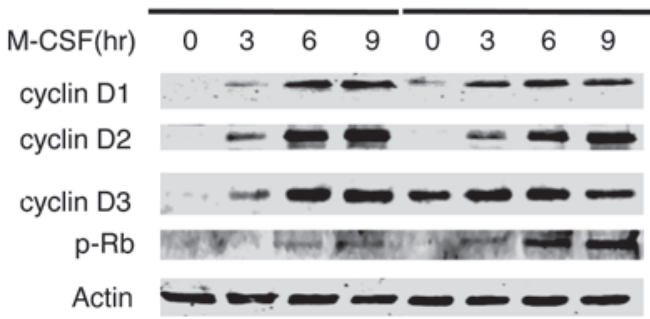

$\mathbf{E}$

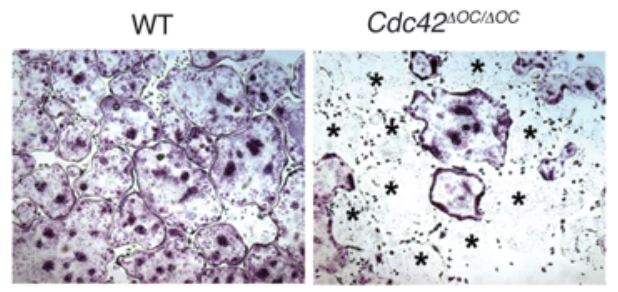

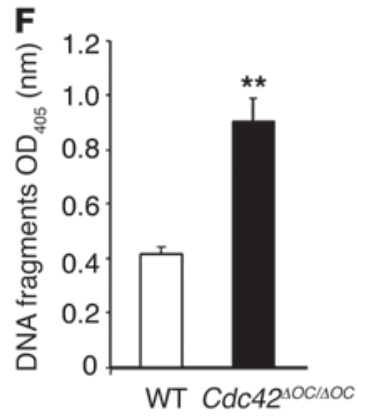

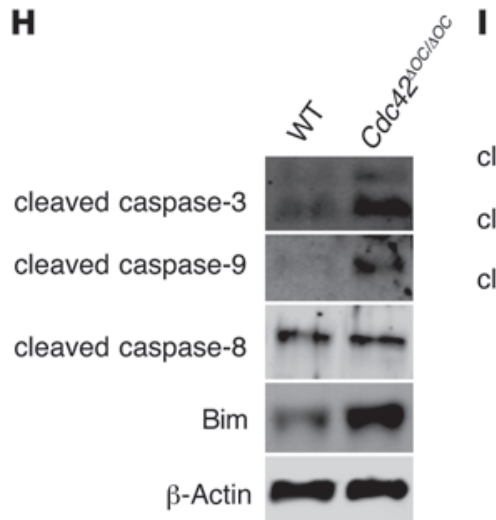

I

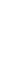

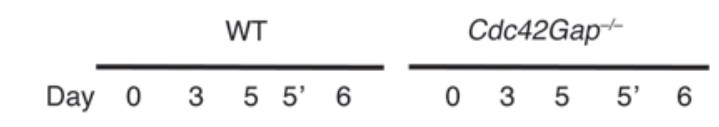

cleaved caspase-3

cleaved caspase- 9

cleaved caspase-8

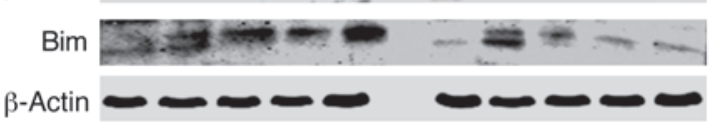

Figure 3

Cdc42 regulates proliferation and survival of osteoclast lineage cells. (A) BrdU incorporation of WT, Cdc42--/, and Cdc42-- rescued with WT Cdc42 BMMs exposed to M-CSF (50 ng/ml) for 12 hours. (B) BrdU incorporation by WT, Cdc42Gap-/, Cdc42Gap - $^{-/}$transduced with WT, or GAP-deficient mutant Cdc42GAPR305A BMMs exposed to M-CSF $(50 \mathrm{ng} / \mathrm{ml})$ for 12 hours. (C and D) Immunoblots of D-type cyclins and phosphorylation of retinoblastoma protein $(\mathrm{Rb})$ in response to M-CSF. $\beta$-Actin served as a loading control. (E) TRAP staining of WT and Cdc $42^{\triangle O O C / \triangle O C}$ osteoclasts. Apoptotic osteoclasts are indicated with asterisks. Original magnification, $\times 200$. (F and $\mathbf{G})$ ELISA-determined osteoclast apoptosis. (H and I) Immunoblots of cleaved caspase-3, -9 , and -8 and Bim in mature WT, Cdc $42^{\Delta O C / \triangle O C}$, and Cdc42Gap ${ }^{-1-}$ osteoclasts. $5^{\prime}$ represents mature osteoclasts deprived of cytokines and serum for 3 hours prior to assay. $\beta$-Actin served as a loading control. Data are presented as mean \pm SD $\left({ }^{* *} P<0.01 ;{ }^{* * *} P<0.001\right)$.

we used loxP/Cre technology to produce a loss-of-function model. Thus, we crossed floxed Cdc42 and cathepsin K-Cre knockin mice $\left(\mathrm{Cts} \mathrm{K}^{\mathrm{Cre} /}+\right)$, thereby targeting the $\mathrm{Cdc} 42$ gene only in cells committed to the osteoclast phenotype $(16,17)$. The progeny of $\mathrm{Cdc}_{\mathrm{C} 2}{ }^{+/+} \mathrm{Cts} \mathrm{K}^{\mathrm{Cr} / /+}$ and $C d c 42^{f / f l} \mathrm{Cts} \mathrm{K}^{\mathrm{Cre} /}$ breeding pairs were designated $\mathrm{Cdc} 42 \mathrm{WT}$ and $\mathrm{Cdc} 42$ osteoclast conditional knockout $\left(C d c 42^{\triangle O C} / \Delta O C\right)$, respectively. Excision of the $C d c 42$ gene was confirmed by PCR in cultured BM macrophages (BMMs), tartrate-resistant acid phosphataseexpressing (TRAP-expressing) mononuclear cells treated 3 days with RANKL and M-CSF (pre-osteoclasts), and mature osteoclasts generated by exposure to the cytokines for 5 days. Specifically, the floxed 762-bp fragment disappeared progressively beginning on day 3 (Supplemental Figure 1A; supplemental material available online with this article; doi:10.1172/JCI39650DS1), as did the Cdc42 protein (Supplemental Figure 1B).

GAPs stimulate intrinsic GTPase activity of Rho GTPases, thus inactivating them. Hence, Cdc42Gap-deficient cells are hyperresponsive to GTPase agonists and represent gain-of-function models. On the other hand, most mice in whom Cdc42GAP is globally deleted die before weaning and those surviving display severe growth retardation (18). We therefore produced BM chi-

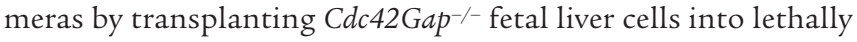


A

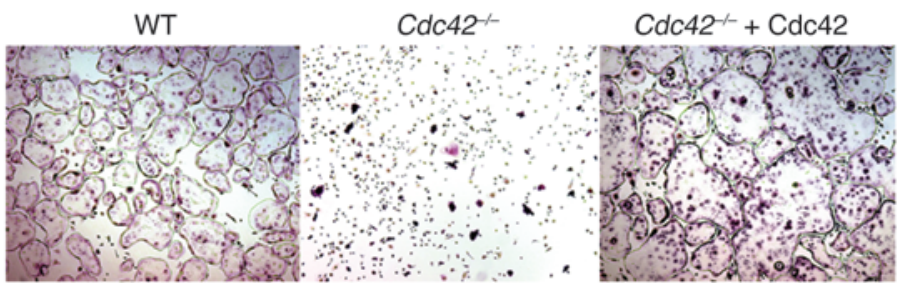

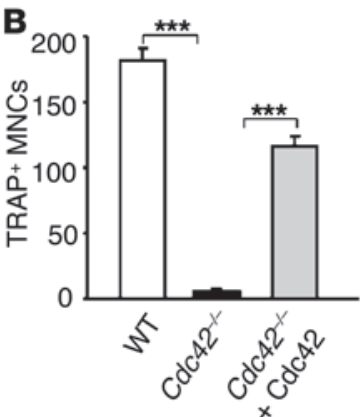

C

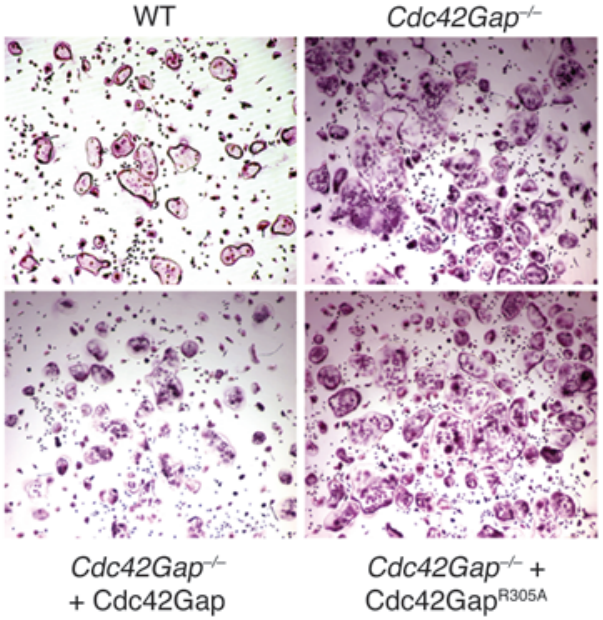

D

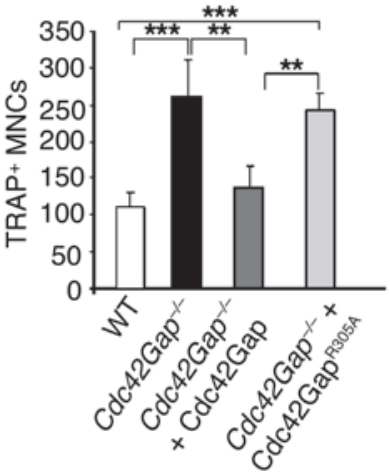

$\mathbf{E}$

$\frac{\text { WT }}{\text { BMM pOC OC BMM pOC OC }} \frac{\text { Cdc42- }}{\text { BMM pOC OC }} \frac{\text { CdC42Gap }}{\text { BMM pOC OC }}$

cathepsin $\mathrm{K}$

\begin{tabular}{llll}
\hline 1.0 & 0.9 & 0.6 & 0.6 \\
\hline & - &
\end{tabular}

\begin{tabular}{llll}
\hline 1.0 & $\overline{5.5}$ & $-\overline{3.8}$ & 4.8
\end{tabular}

MMP-9

$\begin{array}{llll}-1.0 & -0.8 & 0.6 & 0.6\end{array}$

\begin{tabular}{llll}
1.0 & 5.5 & 3.8 & 4.8 \\
\hline 1.0 & 4.7 & 3.1 & 5.2
\end{tabular}

$\beta_{3}$-integrin

\begin{tabular}{|cccc}
1.0 & 0.8 & 0.6 & 0.6 \\
\hline & - & & \\
\hline 1.0 & 0.8 & 0 & 0 \\
\hline & - \\
\hline
\end{tabular}

\begin{tabular}{ll}
$1.0 \quad 4.7$ \\
\hline
\end{tabular}

$3.2 \quad 5.2$

GAPDH

-....

Cdc42

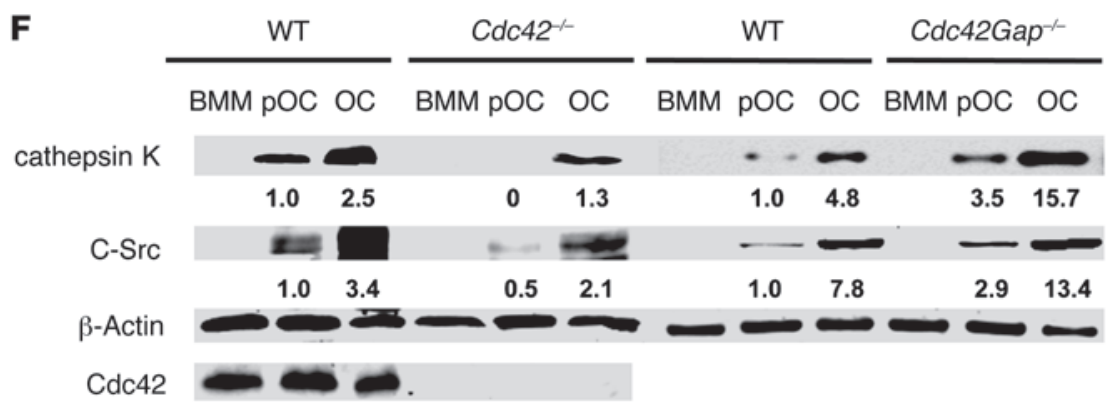

G

\begin{tabular}{|c|c|c|c|c|c|c|c|c|c|c|c|c|c|c|}
\hline \multirow[b]{2}{*}{ Day } & \multicolumn{4}{|c|}{ WT } & \multicolumn{3}{|c|}{$\mathrm{Cdc} 42^{--}$} & \multirow{2}{*}{$\begin{array}{l}\mathbf{H} \\
\operatorname{RANKL}(\mathrm{min})\end{array}$} & \multicolumn{3}{|c|}{ WT } & \multicolumn{3}{|c|}{$\mathrm{Cdc}_{2} \mathrm{Gap}^{--}$} \\
\hline & 0 & 1 & 2 & 30 & 1 & 2 & 3 & & 0 & 5 & 15 & 0 & 5 & 15 \\
\hline JFATC & E. & & & - & & & $\ldots$ & P-MITF & 2 & - & - & - & - & $=$ \\
\hline
\end{tabular}

Figure 4

Cdc42 regulates osteoclast differentiation in vitro. (A) TRAP staining of osteoclasts generated from WT and $\mathrm{Cdc} 42^{-/-}$BMMs produced by transducing $\mathrm{Cdc} 42^{\text {fl/fl }} \mathrm{BMMs}$ with retrovirus expressing Cre recombinase, and Cdc42--- BMMs rescued with WT Cdc42. Original magnification, $\times 200$. (B) Quantification of the number of TRAP+ multinucleated osteoclasts (MNCs) shown in A. (C) TRAP staining of osteoclasts generated from WT or $\mathrm{Cdc} 42 \mathrm{Gap}^{-/}$BMMs or Cdc42Gap ${ }^{-1-}$ BMMs transduced with either WT or the GAP-deficient mutant, Cdc42Gap ${ }^{\mathrm{R} 305 \mathrm{~A}}$. Original magnification, $\times 40$. (D) Quantification of results from C. (E) Osteoclast (OC) differentiation markers assessed by RT-PCR. Numbers represent densitometrically determined ratios relative to GAPDH. (F) Osteoclast differentiation markers assessed by immunoblot. Numbers represent densitometrically determined ratios relative to $\beta$-actin. (G) Induction of NFATc1 protein during osteoclast differentiation of WT and $\mathrm{Cdc} 42^{-/-}$BMMs. $\beta$-Actin served as loading control. (H) MITF phosphorylation with time of exposure to RANKL. $\beta$-Actin served as loading control. Data shown in $\mathbf{B}$ and $\mathbf{D}$ are presented as mean \pm SD ( $\left.{ }^{\star \star} P<0.01 ;{ }^{* \star *} P<0.001\right)$. 
A

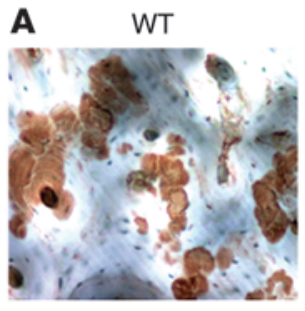

Cdc42--

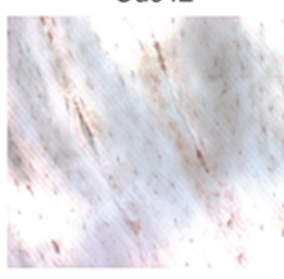

$\mathrm{Cdc}_{2} 2^{-}+\mathrm{Cdc} 42$

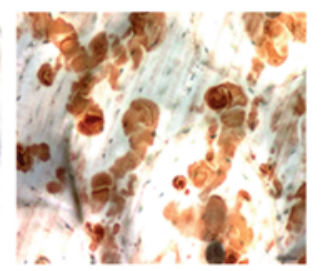

B

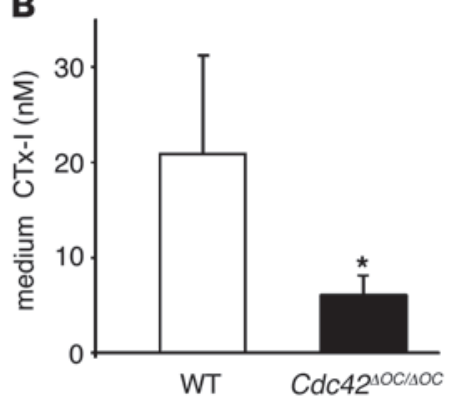

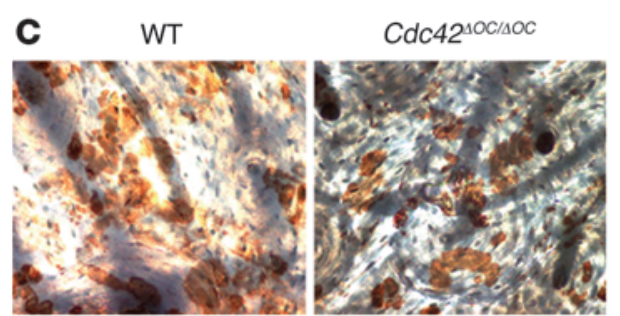
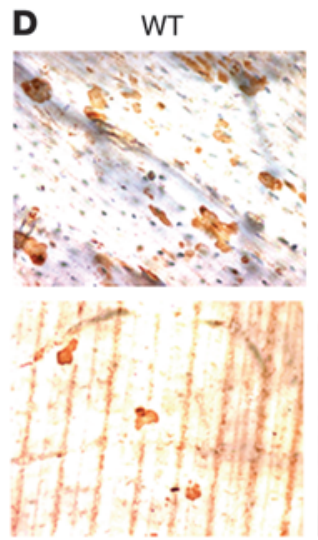

Cdc42Gap ${ }^{-1-}$

+ Cdc42Gap

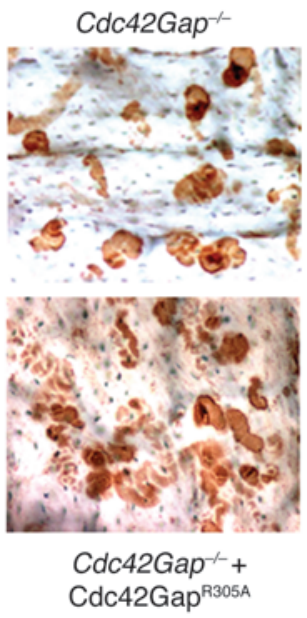

E

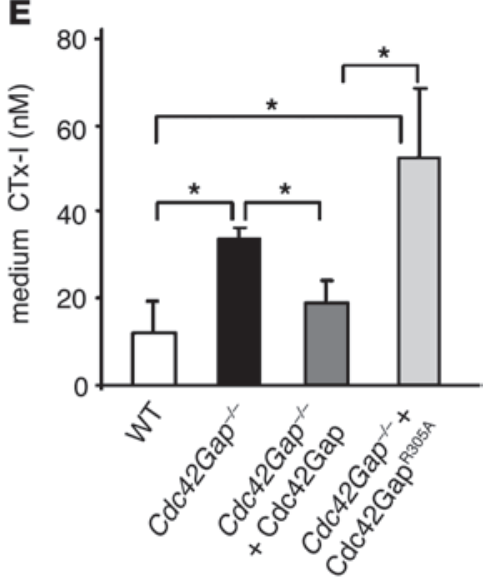

Figure 5

Cdc42 promotes bone resorption in vitro. (A, C , and D) Bone resorption pits labeled by lectin (brown reaction product). Original magnification, $\times 200$. (B and E) Culture medium CTx-1 determined by ELISA. Data are presented as mean \pm SD $\left({ }^{*} P<0.05\right)$.

irradiated WT recipients. WT fetal liver cells, injected into similar hosts, served as a positive control. The genotype of isolated BMMs was determined by PCR (Supplemental Figure 1C). BMMs isolated from $\mathrm{Cdc} 42 \mathrm{Gap}^{-/-}$chimeric mice were hyperresponsive to $\mathrm{M}$-CSF, as evidenced by increased abundance of the GTP-associated GTPase (Supplemental Figure 1D). In contrast, the activation state of Rac, a related GTPase, was unaltered, confirming specificity of Cdc42Gap.

Cdc42 controls $M-C S F$ and RANKL signaling. To determine whether Cdc42 regulates M-CSF or RANKL signaling in osteoclastic cells, we examined all known major effectors of the 2 cytokines in our gain- and loss-of-function models. The small GTPase was required for M-CSF activation of $\mathrm{p} 38$ and the Akt/GSK3 $\beta$ axis but not ERKs (Figure 2, A and B). RANKL activation of JNK, p38, ERK, and Akt was also controlled by Cdc42, which, however, did not affect NF-кB (Figure 2, C and D). Thus, Cdc42 selectively modulates multiple $\mathrm{M}-\mathrm{CSF} / \mathrm{RANKL}$-induced osteoclastogenic signals.

Cdc42 controls BMM proliferation and osteoclast survival and differentiation in vitro. Having established that $\mathrm{Cdc} 42$ was activated by M-CSF and RANKL in osteoclast precursors, and that the small GTPase modulated c-Fms and RANK signaling, we determined whether established cellular events induced by the cytokines were subject to $\mathrm{Cdc} 42$ regulation, once again using gain- or loss-of-function cells. Genetic crossing of $C d c 42^{f / f l}$ mice with animals expressing one copy of Cre recombinase in the cathepsin $\mathrm{K}$ locus eliminated the gene only in mature polykaryons. To obtain early $\mathrm{Cdc} 42^{-/-}$osteoclast precursors, we transduced $\mathrm{Cdc} 42^{f / f l} \mathrm{BMMs}$ with Cre-containing retrovirus (19). WT cells similarly transduced served as a positive control. Providing additional proof of specificity, we performed

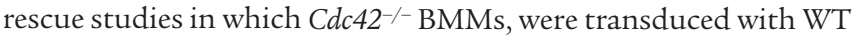
Cdc42. To obtain Cdc42 gain-of-function osteoclast lineage cells, we isolated $\mathrm{BMMs}$ from $\mathrm{Cdc} 42 \mathrm{Gap}^{-/-}$radiation chimera mice. Controls included $\mathrm{Cdc} 42 \mathrm{Gap}^{-/-} \mathrm{BMMs}$ transduced with retroviruses bearing either WT Cdc42Gap or a mutant construct lacking GAP activity (Cdc42Gap ${ }^{R 305 A}$; ref. 20). BMMs lacking or expressing WT $\mathrm{Cdc} 42$ and Cdc42Gap or the mutated form of the latter were differentiated into osteoclasts in M-CSF and RANKL.

Osteoclast number is influenced by precursor propagation and apoptosis of precursors and/or mature cells. Establishing compromised proliferation, BrdU incorporation was decreased in Cdc42-/- BMMs treated with M-CSF and rescued by reconstitution with WT Cdc42 (Figure 3A). Conversely, Cdc42Gap-null BMMs were more proliferative and normalized by WT but not mutant Cdc42Gap (Figure 3B).

We previously established that M-CSF promotes proliferation of primary macrophages by activating D-type cyclins and their downstream effector, hyperphosphorylated $\mathrm{Rb}$ (21). Thus, we measured these cell cycling molecules in the context of Cdc42 loss and gain of function. Phosphorylation of Rb and cyclin D isoforms 1-3 were induced in both genotypes in response to M-CSF, but the changes were more modest in the absence of Cdc42 (Figure 3C). Furthermore, the increased sensitivity of $\mathrm{Cdc} 42 \mathrm{Gap}^{-/-} \mathrm{BMMs}$ to M-CSF (Supplemental Figure 1D) resulted in more cyclin D1 and $\mathrm{Rb}$ phosphorylation in response to the cytokine (Figure 3D). 
A

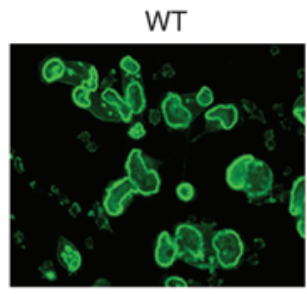

Cdc42--

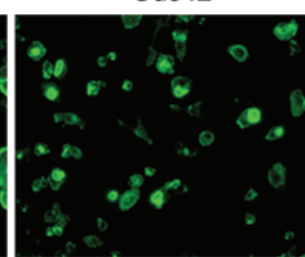

Cdc42 - + Cdc42

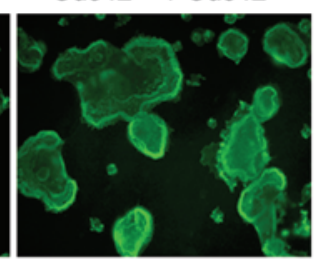

C

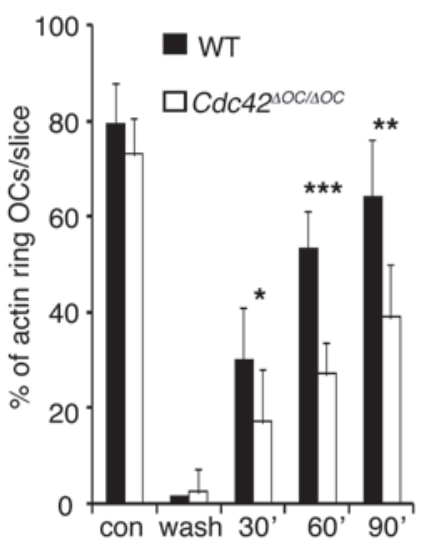

D

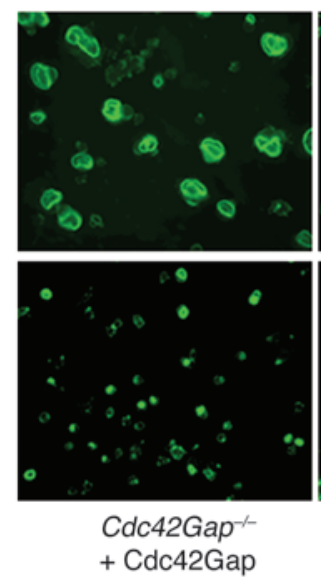

Cdc42Gap ${ }^{-1-}$

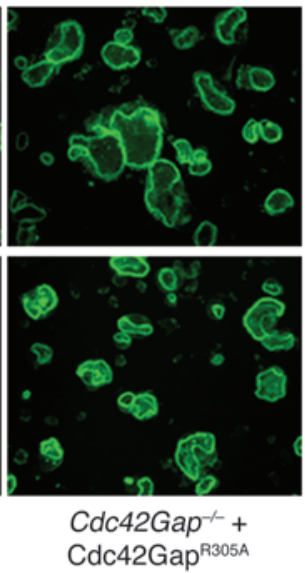

B

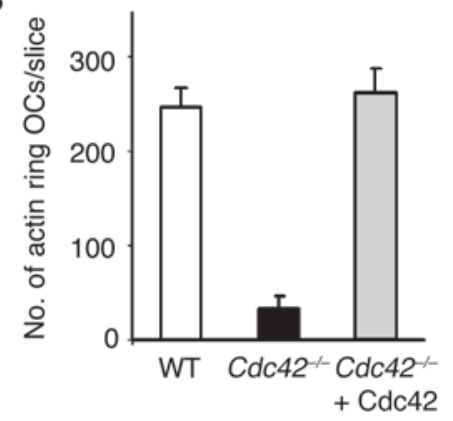

E

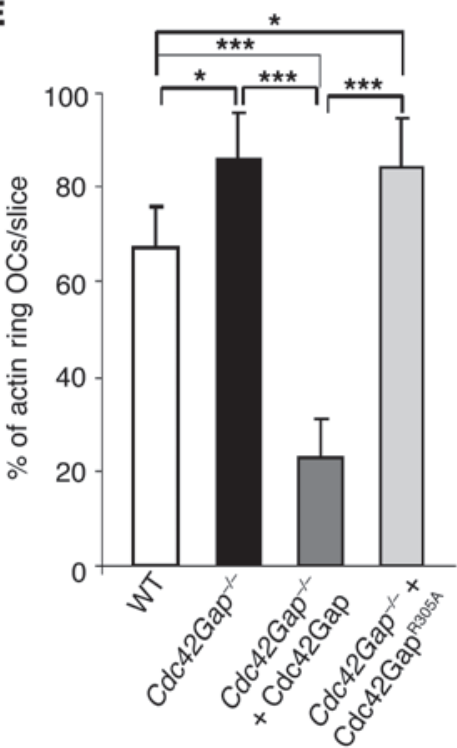

Figure 6

Cdc42 regulates actin ring kinetics. (A and D) F-actin staining in osteoclasts generated on bone slices demonstrated actin rings in all panels. ( $\mathbf{B}$ and $\mathbf{E}$ ) Quantification of the number of actin ring-containing osteoclasts illustrated in $\mathbf{A}$ and $\mathbf{D}$, respectively. (C) Actin ring re-formation assay

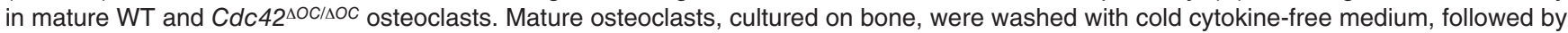
incubation at $37^{\circ} \mathrm{C}$ for 30 minutes. RANKL $(100 \mathrm{ng} / \mathrm{ml})$ was then added for the indicated time. Bone slices were fixed, and F-actin was stained with Alexa Fluor 488-phalloidin. The number of total osteoclasts and those with actin rings were counted. Data are presented as mean \pm SD $\left({ }^{\star} P<0.05 ;{ }^{* *} P<0.01 ;{ }^{* *} P<0.001\right)$.

In contrast to proliferation, apoptosis of WT, $C d c 42^{\triangle O C / \Delta O C}$, and

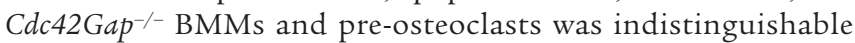
in vitro (data not shown). However, mature osteoclasts lacking Cdc42 died more rapidly than WT upon withdrawal of M-CSF and RANKL (Figure 3, E and F). Consistent with these observations, survival of $\mathrm{Cdc} 42 \mathrm{Gap}^{-1-}$ osteoclasts was prolonged (Figure 3G).

Apoptosis is induced via the intrinsic and/or extrinsic pathways. In the former, pro-apoptotic Bcl-2 family proteins promote cytochrome $\mathrm{c}$ release from mitochondria, and consequently, activation of caspase-9 and caspase-3. In the death receptor scheme, ligation of specialized receptors such as Fas activates initiator caspase-8 and ultimately, caspase-3 (22). To determine which pathway is involved in Cdc42-regulated apoptosis, we assessed the activity of caspases-8, -9 , and -3 and the quantity of Bim, a pro-apoptotic Bcl-2 family member functional in osteoclasts (23). After 3 hours of cytokine and serum starvation, $C d c 42^{\triangle O C / \triangle O C}$ osteoclasts, generated by 5 days in M-CSF and RANKL, contained more Bim and activated caspases-9 and -3 than WT cells (Figure 3H). In keeping with their resistance to apoptosis, $\mathrm{Cdc} 42 \mathrm{Gap}^{-/-}$osteoclast lineage cells produced a paucity of caspase-3 or activated caspase-9 (Figure 3I). In contrast to cas- pase- 9 , altered activation of caspase- 8 was not detectably induced by either loss or gain of function of $\mathrm{Cdc} 42$. This in vitro role of $\mathrm{Cdc} 42$ in osteoclast apoptosis mirrors that occurring in vivo (see below).

The enhanced rate of apoptosis and inhibited precursor proliferation diminishes the abundance of mature polykaryons in

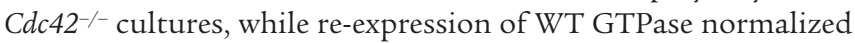
the process (Figure 4, A and B). Alternatively, $\mathrm{Cdc} 42 \mathrm{Gap}^{-/-} \mathrm{BMMs}$ yielded increased numbers of osteoclasts, and the phenotype was reversed by re-expression of WT Cdc42Gap but not the GAP-deficient mutant (Figure 4, C and D). As confirmation that the small GTPase controls osteoclast number via its effects on maturation, expression of osteoclast mRNA (Figure 4E) and protein (Figure 4F) maturation markers was decreased in $\mathrm{Cdc}_{\mathrm{C} 2^{-/}}$precursors, while the same parameters were upregulated in $\mathrm{Cdc} 42 \mathrm{Gap}^{-/-}$cells. To explore further the role of $\mathrm{Cdc} 42$ in osteoclast differentiation, we examined NFATc1 and MITF, 2 transcription factors that regulate the process. While NFATc1 was reduced in the absence of Cdc42, RANKLinduced phosphorylation of MITF, which activates this molecule (24), was enhanced in Cdc42Gap ${ }^{-/-}$cells (Figure 4, G and H). Thus, Cdc 42 promotes osteoclast differentiation. 


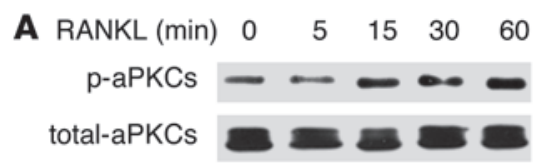

B

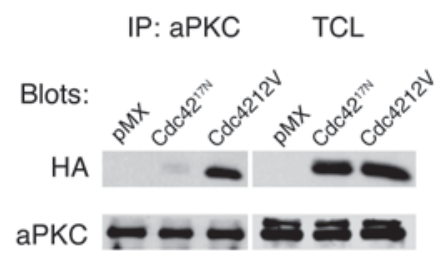

D

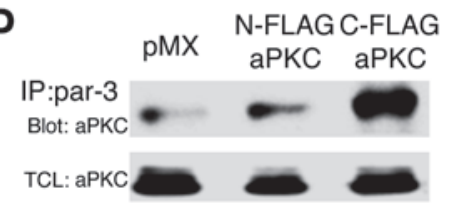

$\mathbf{F}$
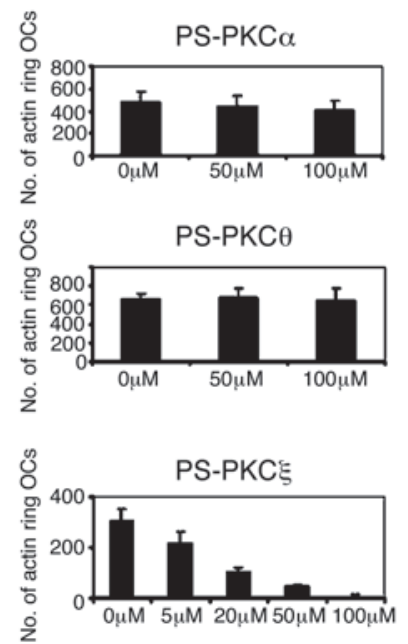

$\begin{array}{llll}0 & 5 & 15 & 30\end{array}$

IP: anti-p-Y Blot: $a$ PKC IP: $a P K C$ Blot: aPKC

C

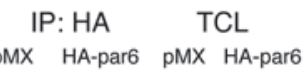
Blots:

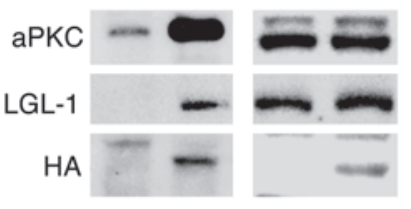

E
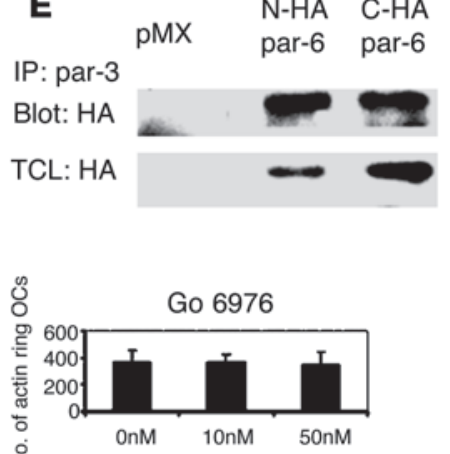

ํ.

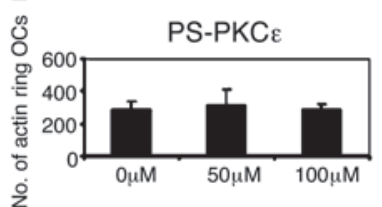
ż

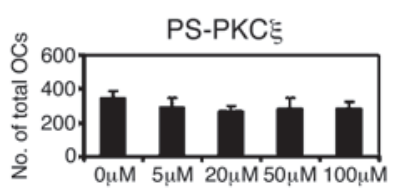

G
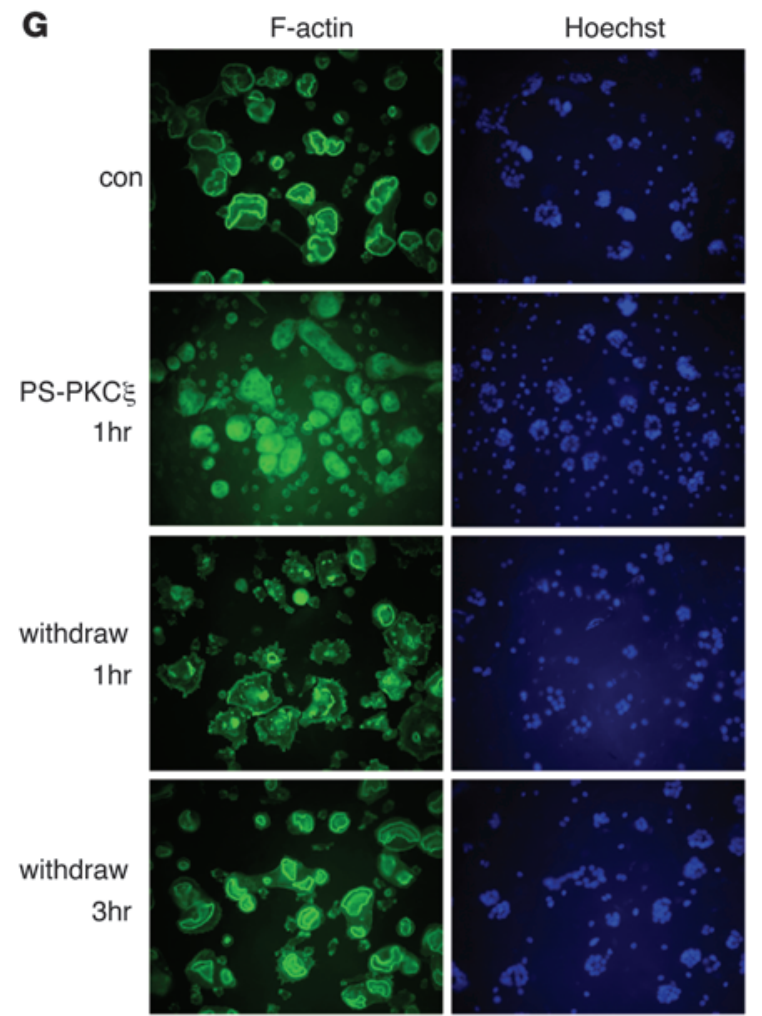

H

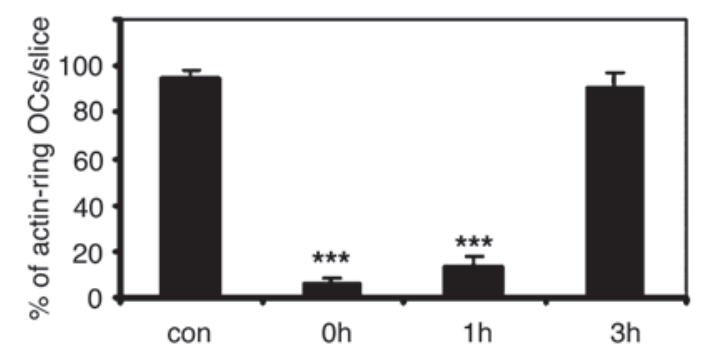

Figure 7

Par-3/Par-6/aPKCs complex mediates Cdc42-regulated osteoclast polarization. (A) RANKL stimulates threonine and tyrosine phosphorylation of aPKCs in mature osteoclasts. Mature osteoclasts, generated by 5 days' exposure of WT BMMs to RANKL and M-CSF, were serum- and cytokine-starved and then treated with RANKL over time. Left: Lysates were immunoblotted for threonine-phosphorylated and total aPKC. Right: Lysates were immunoprecipitated for total phosphotyrosine ( $\mathrm{p}-\mathrm{Y}$ ) using mAb 4G10 or aPKC. Immunoprecipitates were immunoblotted for aPKC. (B) Immunoprecipitation of endogenous aPKCs in osteoclasts transduced with empty vector (pMX), HA-tagged dominant-negative (Cdc42-17N), or HA-tagged constitutively active (Cdc42-12V) retroviruses. TCL: total cell lysates. (C) Co-immunoprecipitation of HA-tagged Par-6 with aPKCs and LGL in osteoclasts. (D) Co-immunoprecipitation of endogenous Par-3 in osteoclasts transduced with pMX, N-terminal-FLAG-tagged PKC- $\lambda$ (N-FLAG-aPKC), or C-terminal-FLAG-tagged PKC- $\lambda$ (C-FLAG-aPKC). (E) Co-immunoprecipitation of endogenous Par-3 in osteoclasts transduced with N-terminal HA-tagged par-6 (N-HA par-6) or C-terminal HA-tagged par-6 (C-HA par-6). (F) Selective inhibition of PKC isoforms in mature osteoclasts, cultured on bone slices, by 1 hour exposure to cell-permeable pseudo substrate (PS) peptide inhibitors of PKC- $\alpha$, PKC- $\theta$, PKC- $\varepsilon$, PKC- $\zeta$, and Go 6976. (G) Osteoclasts were incubated with PKC- $\zeta$ inhibitor or carrier for 1 hour followed by washing and cultured for 1 or 3 hours. Cells were stained to visualize actin rings. Hoechst stain visualizes nuclei. Original magnification, $\times 200$. (H) Quantification of the percentage of osteoclasts exhibiting actin rings illustrated in $\mathbf{F}\left({ }^{* \star *} P<0.001\right)$.

Cdc42 regulates osteoclast function in vitro. While our data indicate that Cdc42 controls osteoclast differentiation, these experiments provide no insights into the role of the small GTPase in the resorptive capacity of the cell. To address this issue, we generated osteoclasts on dentin slices using $C d c 42^{-/-}$and Cdc42GAP precursors as well as their retrovirally rescued counterparts. We visualized resorption lacunae by staining with wheat germ lectin conjugated to horseradish peroxidase and determined global bone degradation as a function of CTx-1 fragments released into culture medium. In keeping with their failure to generate mature osteoclasts, $C d c 42^{---}$BMMs treated for 5 days with RANKL and M-CSF were unable to resorb bone, but the defect was rescued by retroviral reconstitution of the GTPase (Figure 5A). Alternatively, $C d c 42^{\triangle O C / \triangle O C}$ osteoclasts, which differentiate normally, formed 
A

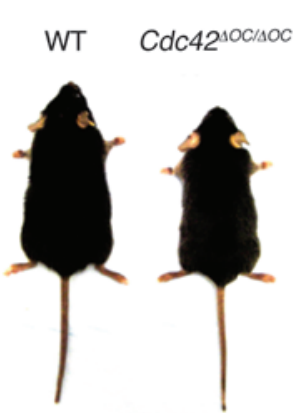

C

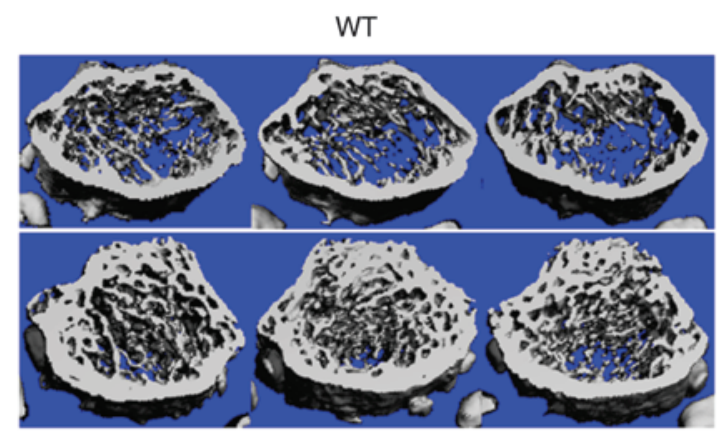

Cdc42 $100 / 100$

B

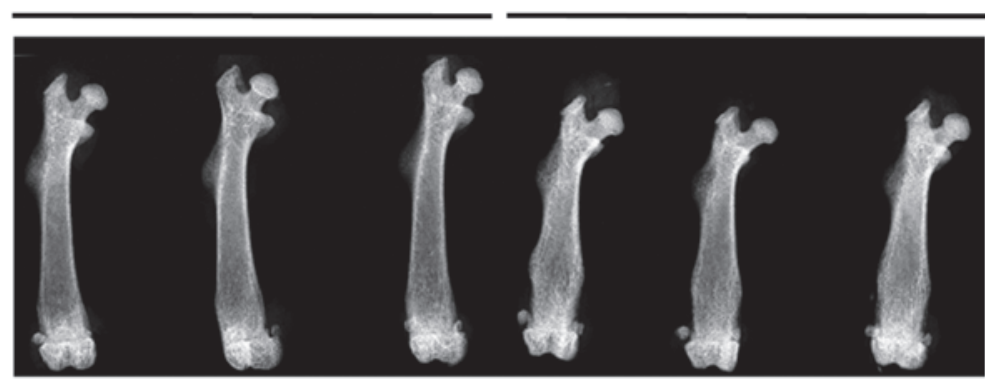

D

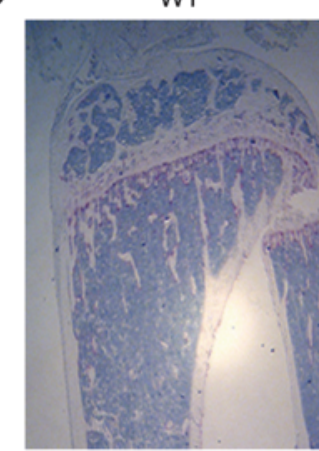

$C d c 42^{100 / 100}$

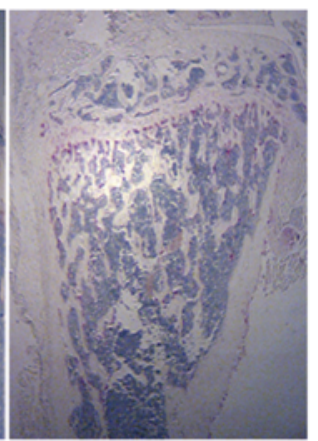

E
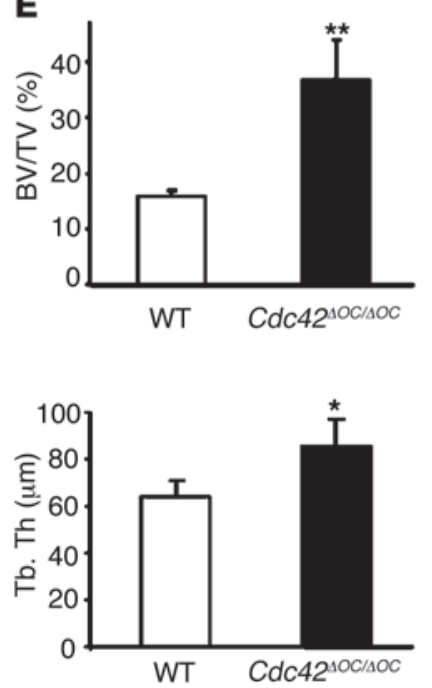

H

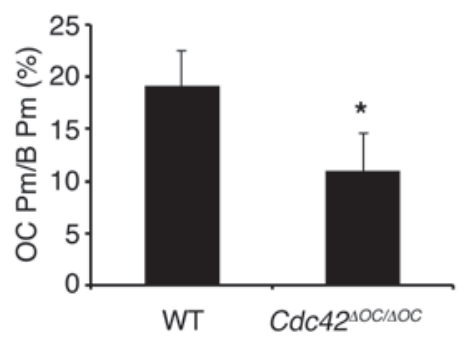

F
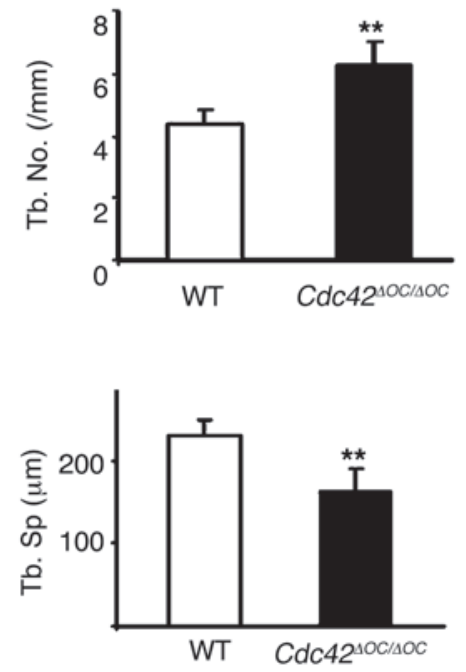

I
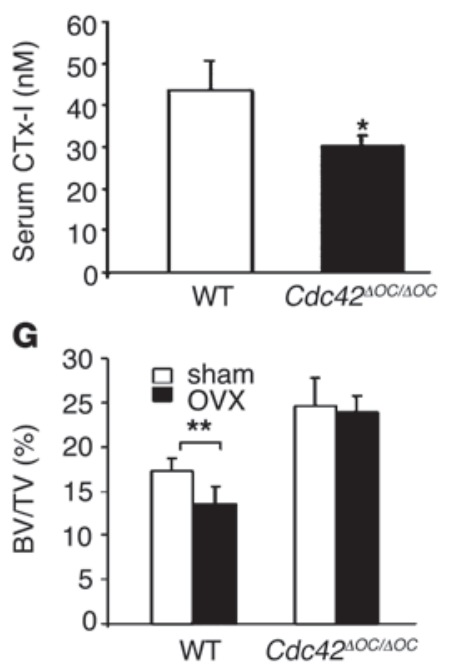

J
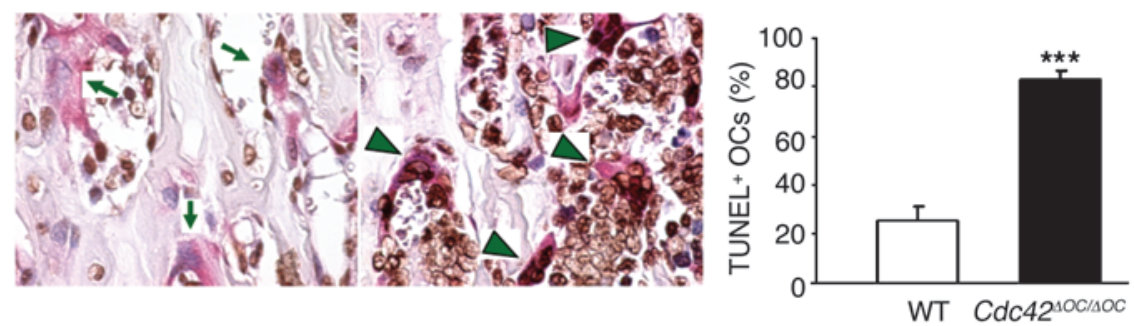


\section{Figure 8}

Increased bone mass in $C d c 42^{1 O C / \triangle O C}$ mice due to suppressed resorption. (A) WT and $C d c 42^{\triangle O C / \triangle O C}$ mice. (B) Radiographs of femurs from 8-week-

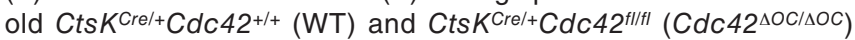
male mice. (C) $\mu \mathrm{CT}$ images of distal femoral metaphysis of WT and $C d c 42^{\triangle O C / \triangle O C}$ mice. (D) TRAP-stained histological sections of proximal tibia of WT and $C d c 42^{\triangle O C / \triangle O C}$ mice $(n=10)$. Original magnification, $\times 40$. (E) $\mu \mathrm{CT}$ determination of BV/TV, trabecular number (Tb. No), trabecular thickness (Tb. Th), and trabecular separation (Tb. Sp) of distal femoral metaphysis. (F) Serum CTX-1 of WT and Cdc42 ${ }^{\triangle O C / \Delta O C}$ mice. (G) $\mu \mathrm{CT}$ determination of BV/TV of distal femoral metaphysis from 10 sham-operated (sham) or ovariectomized 12-week-old WT and $\mathrm{Cdc} 42^{\mathrm{AOC} / \triangle O C}$ mice 4 weeks after surgery. (H) WT and $C d c 42^{\triangle O C / \triangle O C}$ tibiae were stained for TRAP activity, and the percentage of bone surface in juxtaposition to osteoclasts was histomorphometrically determined. (I) Double staining of TUNEL and TRAP in histological sections of proximal tibia. TRAP+ multinucleated osteoclasts are indicated by arrows, and those staining for both TRAP and TUNEL are indicated by arrowheads. Original magnification, $\times 400$. (J) Percentage of TUNELpositive osteoclasts shown in I $\left({ }^{*} P<0.05 ;{ }^{* \star} P<0.01\right.$; $\left.{ }^{\star \star \star} P<0.001\right)$.

pits, but the magnitude of bone resorption, reflected by medium CTx-1, was diminished (Figure 5, B and C). While this observation suggests compromised osteoclast function in mature osteoclasts lacking $\mathrm{Cdc} 42$, it must be viewed in the context of the accelerated apoptosis experienced by these cells. On the other hand, the fact that the percentage of $C d c 42^{\triangle O C / \triangle O C}$ osteoclasts capable of forming actin rings is diminished speaks to impaired bone resorption on a per-cell basis (see below). In contrast to absence of the GTPase, increased Cdc42 activity in osteoclasts lacking Cdc42Gap enhanced resorption, which was normalized by retroviral expression of the deleted WT protein but not R305A mutant in which GAP activity was disrupted (Figure 5, D and E).

Osteoclast function depends upon the actin cytoskeleton, which in other cells is organized by Cdc42 (25). In this regard, the number of actin rings in cultures of $\mathrm{Cdc} 42^{-/-}$osteoclasts was reduced by approximately $80 \%$ and rescued by retroviral Cdc 42 reconstitution (Figure $6, \mathrm{~A}$ and $\mathrm{B}$ ). Interpretation of these data is confounded, however, by failure of $\mathrm{Cdc} 42^{-/-}$BMMs to undergo normal osteoclast differentiation. Thus, the reduction in actin ring development may reflect a paucity of resorptive, mutant polykaryons. The small circumference of $\mathrm{Cdc} 42^{-/-}$actin rings is also consistent with the relative abundance of mononuclear cells in these cultures (Figure 6A).

In contrast to $C d c 42^{-/-}$cells, $C d c 42^{\triangle O C / \triangle O C}$ BMMs differentiated normally because the GTPase was absent only in mature osteoclasts (Supplemental Figure 2). We asked, therefore, whether actin ring formation was disturbed in these polykaryons lacking Cdc42. In fact, the percentage of actin ring-containing osteoclasts generated from $C d c 42^{\triangle O C / \triangle O C} B M M s$ was indistinguishable from WT BMMs (data not shown). Given that actin ring turnover is rapid in osteoclasts (26), we hypothesized that although Cdc42 does not influence the ultimate numbers of actin rings, it may govern the rate of their formation. To test this, mature osteoclasts generated on bone were washed in medium at $4{ }^{\circ} \mathrm{C}$ to disrupt the actin rings. The cells were then incubated in cytokine-free medium at $37^{\circ} \mathrm{C}$ for 30 minutes, after which they were exposed to RANKL for 30, 60, or 90 minutes to promote ring re-formation (27). Figure $6 \mathrm{C}$ and Supplemental Figure 3 show that, at all times tested, the rate of RANKL-stimulated actin ring development is retarded in the absence of Cdc42. Further supporting a role of Cdc42 in cytoskeletal organization, actin ring formation by $\mathrm{Cdc} 42 \mathrm{Gap}^{-/-}$osteoclasts was increased, while re-expression in these Cdc42Gap cells, but not in GAP-deficient mutant cells, blunted the process (Figure 6, D and E). Hence, $\mathrm{Cdc} 42$ is dispensable for ultimate formation of actin rings but regulates the rate at which these structures are generated.

Cdc42 regulates osteoclast polarization. Cdc 42 regulation of actin ring formation is reminiscent of its role in generating and maintaining tight junctions in polarized epithelial cells. In this circumstance, the active GTPase stimulates assembly of the Cdc42/Par-3/ Par-6/aPKC polarity complex $(28,29)$. Although bone-residing osteoclasts are polarized, the protein constituents that establish and sustain this polarity are unknown. Current models suggest the presence of 2 polarizing complexes: inactive Par-6/aPKC/LGL (lethal giant larvae) and active Cdc42/Par3/Par6/aPKC $(30,31)$. Binding of GTP-bound Cdc42 to Par6 activates aPKCs, which phosphorylate LGL, leading to its dissociation and replacement with Par3, the key event that triggers polarization. In the context of osteoclasts, RT-PCR and Western blots showed that the cells expressed Par- 3 and Par-6A as well as the aPKCs PKC- $\zeta$, and PKC- $\lambda$ (Supplemental Figure 4). Of particular note, Par3 increases during osteoclast differentiation, suggesting, as in other cells, that it regulates polarity (32). Finally, RANKL activates aPKCs by phosphorylating threonine and tyrosine (Figure 7A) $(33,34)$. We therefore asked whether $\mathrm{Cdc} 42$ participates in the active polarizing complex in osteoclasts. To this end, we generated osteoclasts expressing dominant-negative (Cdc42-17N) or constitutively active (Cdc42-12V) Cdc42. aPKCs bind only the active form of this small GTPases (Figure 7B) despite similar expression of the mutant protein (Figure 7B). Both aPKC and LGL-1 form a complex with Par-6, which does not contain Par-3 (Figure 7C and data not shown). Furthermore, endogenous Par-3 co-immunoprecipitates with FLAG-tagged PKC- $\lambda$ and HA-tagged Par-6, but not with LGL-1 (Figure 7, D and E, and data not shown). These observations suggest the existence of an active Cdc42/Par-3/Par-6/aPKC- $\lambda$ complex in osteoclasts, formation of which is stimulated by RANKL.

The PKC family comprises 3 major subgroups: conventional, novel, and aPKCs. To discriminate between these family members in osteoclast polarization, we used inhibitors that block PKC- $\alpha,-\beta,-\gamma$ (Go6976, conventional), $-\theta,-\varepsilon$ (novel), or $-\lambda,-\zeta$ (atypical). Neither Go6976 nor peptides targeting novel PKCs altered the number of actin rings (Figure 7F) or osteoclast number (data not shown). In contrast, a peptide inhibitor of aPKCs (PS-aPKC) dose dependently blocked actin ring formation (Figure $7 \mathrm{~F}$, lower left panel) without altering osteoclast abundance (Figure 7F, lower right panel). This inhibitory effect of PS-aPKC was not due to toxicity, as osteoclast apoptosis, determined by DNA-fragmentation ELISA, was unaltered (data not shown) and withdrawal of the inhibitor for 3 hours normalized actin ring formation (Figure 7, G and $\mathrm{H}$ ).

$\mathrm{Cdc} 42$ is essential for osteoclastic bone resorption in vivo and its ablation prevents ovariectomy-induced bone loss. Having established that $\mathrm{Cdc} 42$ regulates osteoclast function in vitro, we asked whether the same is true in vivo. Tooth eruption of $C d c 42^{\triangle O C / \triangle O C}$ mice was normal (data not shown), but the animals were smaller than WT mice (Figure 8A). Moreover, their femora were abnormally shaped, which indicated abnormal modeling (Figure 8B). Trabecular volume of the mutant bone was enhanced, as were trabecular number and thickness, and these changes were accompanied by decreased trabecular separation (Figure 8, 

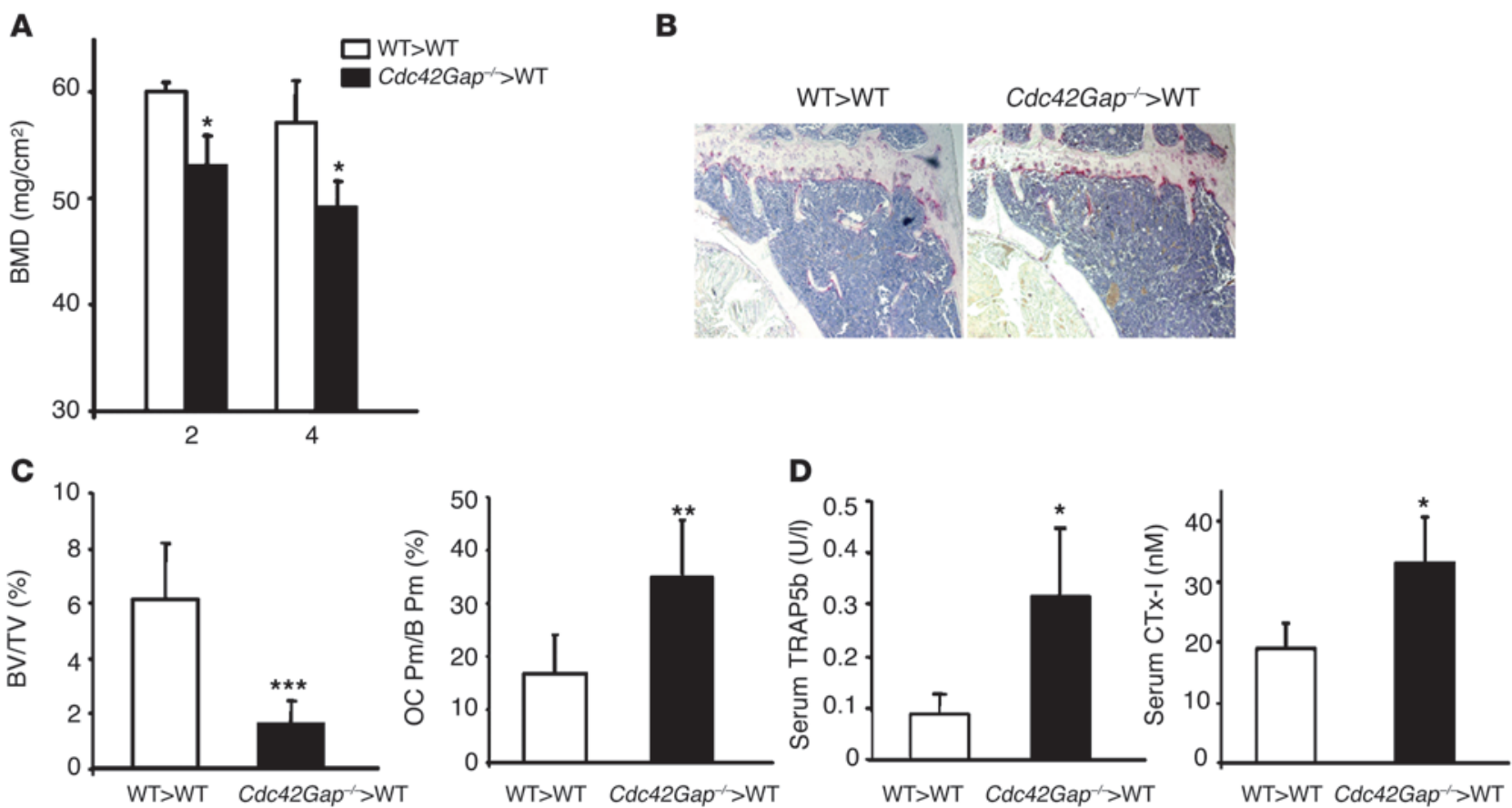

Figure 9

Decreased bone mass in $\mathrm{Cdc} 42 \mathrm{Gap}^{-/}$chimeric mice due to enhanced bone resorption. (A) BMD of lumbar vertebrae measured by DEXA in WT and $\mathrm{Cdc} 42 \mathrm{Gap}^{-1-}$ chimeric mice 2 months and 4 months after marrow transplantation. (B) TRAP-stained histological sections of proximal tibia of WT and Cdc42Gap ${ }^{-1-}$ chimeric mice 4 months after transplantation $(n=10)$. Original magnification, $\times 40$. (C) BV/TV and osteoclast perimeter per bone perimeter (OC Pm/B Pm) calculated from B. (D) Serum TRAP5b and CTx-1 in WT and Cdc42Gap ${ }^{-/-}$chimeric mice 4 months after transplantation, as measured by ELISA. Data are presented as mean $\pm \mathrm{SD}\left({ }^{\star} P<0.05 ;{ }^{\star \star} P<0.01 ;{ }^{* \star} P<0.001\right)$.

$\mathrm{C}$ and $\mathrm{E})$. These phenotypic changes indicated either arrested bone resorption or stimulated osteogenesis. $C d c 42^{\triangle O C / \triangle O C}$ mice, however, had normal circulating osteocalcin (WT $21.1 \pm 14.7$ versus $C d c 42^{\triangle O C / \triangle O C} 20.1 \pm 6.7 \mathrm{ng} / \mathrm{ml}$ ), which indicated that deletion of the GTPase in the osteoclast does not stimulate the osteoblast. Decreased bone resorption in $C d c 42^{\triangle O C / \triangle O C}$ mice in vivo was confirmed by serum CTx-1 (Figure $8 \mathrm{~F}$ ). Importantly, $C d c 42^{\triangle O C / \triangle O C}$ female mice were protected from ovariectomyinduced (OVX-induced) bone loss (Figure 8G).

Retarded bone resorption may reflect increased osteoclast apoptosis and/or diminished osteoclastogenesis. In fact, osteoclast abundance of $C d c 42^{\triangle O C / \triangle O C}$ mice was diminished, and the percentage of osteoclasts exhibiting features of apoptosis increased 4-fold (Figure 8, $\mathrm{H}$ and $\mathrm{J}$ ).

These findings indicate that $\mathrm{Cdc} 42$ is critical for osteoclastic bone resorption. If this is so, $\mathrm{Cdc} 42$ gain-of-function mice should exhibit a reciprocal phenotype. In this regard, bone mineral density (BMD) of lumbar vertebrae of $\mathrm{Cdc} 42 \mathrm{Gap}^{-/-}$marrow chimeras is decreased at 2 and 4 months of age (Figure 9A). Histomorphometric analysis of proximal tibiae of 4-month-old mice showed that $\mathrm{Cdc} 42 \mathrm{Gap}^{-/-}$chimeras had a lower ratio of bone volume to tissue volume (BV/TV) and increased osteoclast surface (Figure 9, B and

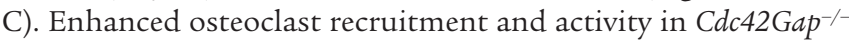
mice were confirmed by increased serum TRAP5b and CTx-1, respectively (Figure 9D).

\section{Discussion}

Our findings, summarized in Figure 10, and based exclusively on the use of genetically manipulated mice and their derived primary cells, indicate what we believe is a novel and multifactorial role for $\mathrm{Cdc} 42$ in osteoclastogenesis and bone resorption in vitro and in vivo. Thus, Cdc42 is crucial for skeletal modeling, remodeling, and bone loss in the context of estrogen withdrawal. The small GTPase exerted its effects by regulating RANKL- and/or M-CSF-dependent precursor proliferation and differentiation, apoptosis of the mature osteoclasts, and their capacity to polarize.

Activated Cdc42, binding to specific effectors, modulates cytoskeleton organization, proliferation, apoptosis, matrix adhesion, polarization, membrane trafficking, and transcription (35). As each of these events is involved in osteoclast differentiation and function, we hypothesized that $\mathrm{Cdc} 42$ participates in the bone resorptive process. While the GTPase is known to be RANKL-activated in osteoclasts (15), most observations to date represent in vitro overexpression of dominant mutants and are contradictory $(7,8)$. Therefore, the significance of $\mathrm{Cdc} 42$ in osteoclast biology remains unresolved. By using genetic models of Cdc42 loss and gain of function, we established that the GTPase broadly regulates the osteoclast by previously uncharacterized means.

Cdc42, activated by M-CSF and RANKL, enhanced BMM proliferation and osteoclast differentiation and survival in vitro and in vivo. Loss or gain of Cdc42 function selectively controlled RANKL stimulation of MAP kinases and Akt but not NF-кB. Similarly, upon c-Fms occupancy, the GTPase activates p38 and Akt but not ERKs. Consistent with our previous findings (21), downregulation of Akt/GSK-3 $\beta$ consequent to absent Cdc 42 decreases D-type cyclins, phosphorylated $\mathrm{Rb}$, and cell proliferation. Hence, the Akt/GSK-3 $\beta / \mathrm{D}$ cyclin-Rb axis mediates Cdc42-regulated osteoclast precursor proliferation. 


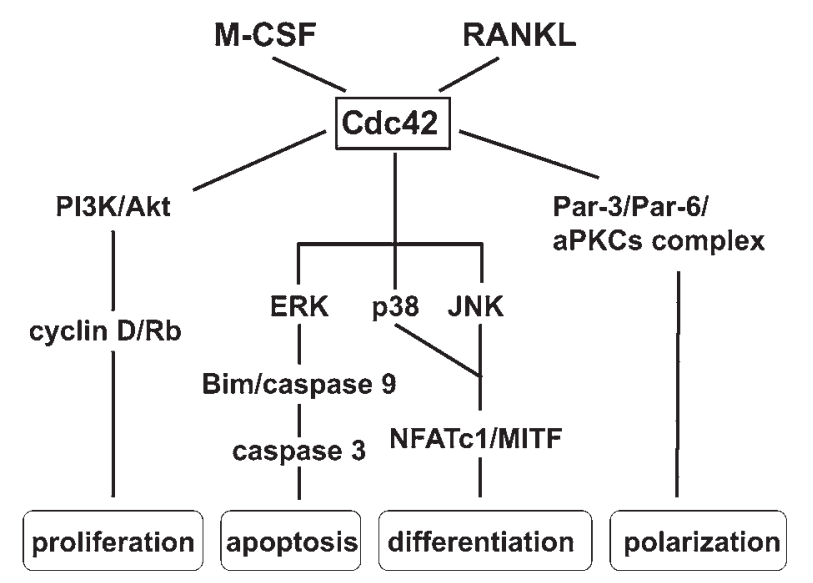

Figure 10

Model of Cdc42-mediated regulation of osteoclast lineage cell proliferation, apoptosis, differentiation, and function.

Apoptosis is another key mechanism controlling bone resorption, as extrinsic death receptor and intrinsic stress pathways attenuate osteoclast survival. For example, Fas/Fas ligand participates in estrogen-induced osteoclast apoptosis in both an autocrine and paracrine manner and is involved in the pathogenesis of postmenopausal osteoporosis $(17,36)$. Mice with defective Fas genes or a Fas ligand mutation have abundant osteoclasts and decreased bone mass (37). Unoccupied $\alpha v \beta 3$ integrin, which mediates osteoclast adhesion to bone, also promotes osteoclast apoptosis by directly activating caspase- 8 and, thus, the extrinsic pathway (38). Alternatively, ERKs and the proapoptotic BH3only Bcl-2 family member, Bim, regulate cell death through the intrinsic pathway (23).

We demonstrated that absence of Cdc42 accelerated osteoclast apoptosis, whereas excess Cdc42-GTP exerted a protective role. In other cells Pak4, a Cdc42 target, blunts apoptosis by promoting phosphorylation and, hence, inactivation of the pro-apoptotic protein $\mathrm{Bad}$ (39). We found that $\mathrm{Bim}$, another pro-apoptotic $\mathrm{Bcl}-2$ family member and regulator of the osteoclast (23), was enhanced in the context of $\mathrm{Cdc} 42$.

$\mathrm{M}$-CSF regulates apoptosis through effectors such as Pak1 that promote survival by modulating expression of the inhibitor of apoptosis (IAP) family member (40). These studies, however, relied on overexpression of constitutively active or dominantnegative proteins and did not reveal linkage to $\mathrm{Cdc} 42$. Activated caspase-8- and Fas ligand-induced apoptosis (data not shown) were indistinguishable among WT and Cdc42 loss- and gain-offunction cells. Therefore, unlike $\alpha v \beta 3, \mathrm{Cdc} 42 \mathrm{did}$ not regulate the extrinsic pathway. Conversely, abundance of Bim and activated caspase- $9 / 3$ paralleled apoptosis, indicating that the intrinsic pathway was responsible.

In addition to modifying proliferation and apoptosis, $\mathrm{Cdc} 42$ controlled osteoclast number, in vivo and in vitro, by directly regulating precursor differentiation. RANKL was required for this process and its targets included the key osteoclastogenic transcription factor NFAT2 (NFATc1). M-CSF also promotes RANKL-induced osteoclast development by activating MITF, a basic helix-loophelix/leucine-zipper transcription factor essential for expression of osteoclast-specific genes $(41,42)$. The fact that RANKL-stimulated NFATc1 is blunted in the absence of Cdc42, while M-CSF induction of MITF phosphorylation and activation is enhanced in Cdc42 gain-of-function cells, indicates that GTPase regulates osteoclast differentiation.

JNK activation by RANKL is a necessary event in the cytokine's induction of NFATc1 (43). Similarly, RANKL or M-CSF phosphorylates p38, which stimulates MITF (41). The cytokines also activated Cdc42, which in turn induced phosphorylation of JNK and p38, indicating that GTPase mediated RANK and M-CSF stimulation of osteoclastogenic genes via selected MAPKs.

$\mathrm{Cdc} 42$ is central to cytoskeletal organization, which in the osteoclast can only be fully evaluated on mineralized substrate $(44,45)$. The major known effectors linking activated Cdc 42 to actin include WASP, IQGAP, and PAKs. The WASP-Arp $2 / 3$ complex plays a critical role in actin ring formation and bone resorption (46-48). Thus, it is surprising that neither the appearance of filopodia (Supplemental Figure 5), an actin-enriched structure induced by Cdc42, nor the abundance of actin rings in osteoclasts were affected by absence of the GTPase, suggesting compensation by either Rac or related molecules (12). Indeed, Wrch1, a Rho GTPase that shares sequence similarities with Rac1 and $\mathrm{Cdc} 42$, regulates actin ring formation, osteoclast adhesion, and migration (49). On the other hand, CTx-1 in culture medium or in mouse blood was reduced in the absence of $\mathrm{Cdc} 42$, establishing its necessary, nonredundant role in the osteoclast in vitro and in vivo.

Despite the presence of actin rings in Cdc42-deficient osteoclasts, the formation rate of these cytoskeletal structures was prolonged. Modulation of the kinetics of actin ring generation by Cdc 42 is likely essential for osteoclast function, as illustrated by substantial changes in medium CTx-1 in loss- and gain-of-function cultures The fact that active but not dominant-negative $\mathrm{Cdc} 42$ associates with aPKCs in osteoclasts suggests that, as in other cells, this complex participates in polarization (48). Thus, Cdc42 governs an array of mechanisms by which RANKL and M-CSF promote osteoclast recruitment and function and presents as a candidate therapeutic target for postmenopausal osteoporosis.

\section{Methods}

Antibodies and reagents. Commercially available antibodies were purchased from the following resources: monoclonal anti-PKC- $\lambda$ and Bim (BD Biosciences); JNK, p-JNK, p38, p-p38, Akt, p-Akt, ERK, p-ERK, IкB, p-IKB, GSK3 $\beta$, p-GSK3 $\beta$, p-Rb (807/811), p-aPKCs, cleaved caspase-3, and cleaved caspase-9 (Cell Signaling Technology); monoclonal antiHA (Covance); rabbit anti-Par-3 and aPKCs (Invitrogen); monoclonal anti-cathepsin-K, 4G10, and rabbit anti-Par-3 (Millipore); monoclonal anti-Cdc42, and rac1 (Pierce Biotechnology Inc.); rabbit anti-cyclin D1, anti-cyclin D2, anti-cyclin D3, anti-NFATc1, anti-caspase-8, and antiaPKCs (Santa Cruz Biotechnology Inc.); and monoclonal anti- $\beta$-actin (Sigma-Aldrich). Anti-p-MITF antibody was provided by M.C. Ostrowski (Ohio State University, Columbus, Ohio, USA). Rabbit anti-LGL1 and anti-LGL2 antibodies were provided by P. Brennwald (University of North Carolina, Chapel Hill, North Carolina, USA). Pseudo substrate peptide inhibitors of PKC- $\alpha$, PKC- $\varepsilon$, PKC- $\zeta$, and Go 6976 were purchased from Biomol. Pseudo substrate peptide inhibitor of PKC- $\theta$ was bought from Biosource. Alexa Fluor 488-conjugated phalloidin was bought from Invitrogen. Peroxidase-conjugated wheat germ agglutinin was obtained from Sigma-Aldrich.

Mice. Generation of $C d c 42 \mathrm{Gap}^{-/-}$and $C d c 42^{f / f l}$ mice has been described $(16,18)$. Ctsk $\mathrm{kre}^{\mathrm{c}+}$ mice were provided by S. Kato (University of Tokyo, Tokyo, Japan) (17). Further details are available in Supplemental Figure 1. All animals were housed in the animal care unit of the Washington University 
School of Medicine and were maintained according to guidelines of the Association for Assessment and Accreditation of Laboratory Animal Care. All animal experimentation was approved by the Animal Studies Committee of the Washington University School of Medicine. After body weight measurements, WT and $C d c 42^{\triangle O C / \triangle O C}$ female mice were anesthetized and underwent bilateral OVX via the dorsal approach. Sham-operated mice had the ovaries exteriorized but not removed. Uterine weight was determined at sacrifice to verify the successful removal of the ovaries.

Generation of $\mathrm{Cdc} 42 \mathrm{Gap}^{-/-} \mathrm{BM}$ chimeras. Six-week-old C57BL/6 mice that had received 13.2 Gy of total body $\gamma$-irradiation were injected via the tail vein with $100 \mu \mathrm{l}$ of PBS containing $2 \times 10^{6}$ fetal liver cells from either Cdc42Gap ${ }^{-/-}$mice or their WT littermates as previously described (50). These mice were used at 8-12 weeks after BM transplantation.

$B M D$ measurement and radiographic imaging. BMD of lumbar vertebrae was measured in vivo in anesthetized mice at 2 months and 4 months after transplantation by dual-energy X-ray absorptiometry using a PIXAI2 bone densitometer (GE Medical System) (51). We obtained radiographs of mouse femur with a Faxitron $\mathrm{x}$-ray system (Faxitron $\mathrm{x}$-ray).

$\mu C T$ analysis. Trabecular volume in the distal femoral metaphysis was measured using a Scanco $\mu$ CT40 scanner (Scanco Medical AG) (52). A threshold of 260 was used for evaluation of scans.

Histological analysis. Histomorphometric analysis was performed on TRAP-stained tibia with Osteomeasure software (Osteomeasure). For double staining of TUNEL and TRAP, paraffin sections were stained for TUNEL using ApopTag Peroxidase In Situ Apoptosis Detection Kit (Millipore) and followed by TRAP staining.

Serum TRAP5b and CTx-1 Assay. Blood was collected under anesthesia. Serum TRAP5b was measured using a mouse-specific ELISA assay (Immuno Diagnostic Systems). CTx-1 concentration was determined with the RatLaps ELISA kit (Nordic Bioscience Diagnostics AS).

Osteoclast culture. Primary BMMs were prepared as described previously (51). To generate osteoclasts, $100 \mathrm{ng} / \mathrm{ml}$ RANKL and 1/100 vol CMG 14-12 culture supernatant (53) were added to $\alpha-10$ medium for 4-5 days. Osteoclasts were stained for TRAP as described by the manufacturer's instructions (Sigma-Aldrich). The staining of actin rings and resorption lacuna was performed as described previously (52).

Retroviral transduction. HA-tagged mouse WT Cdc42GAP, HA-tagged mouse Cdc42GAP ${ }^{\mathrm{R} 305 \mathrm{~A}}$, and T7-tagged mouse WT Cdc42 were cloned into pMX-IRES-bsr retroviral vector. HA-tagged Cre, Cdc42 ${ }^{17 \mathrm{~N}}$, Cdc42 ${ }^{12 \mathrm{v}}$, Par-6, and FLAG-tagged PKC- $\lambda$ were cloned into PMX-IRES-puro vector. Retroviral transduction into BMMs was performed as described previously (54).

RT-PCR. RNA was isolated using RNeasy kits (Qiagen). RT-PCR was performed as described previously (52). The primers for Cdc42 were sense (5'-CCCATCGGAATATGTACCAAC-3') and antisense (5'-AGGCTTCTGTTTGTTCTTGGC- $3^{\prime}$ ). The primers for cathepsin $\mathrm{K}, \mathrm{MMP} 9, \beta 3$-integrin, and GAPDH were described previously (52).

Western blot and immunoprecipitation. For RANKL or M-CSF signaling, macrophages were grown in $\alpha-10$ medium and 1/10 CMG14-12 supernatant for 3 days. Cells were then starved of serum and M-CSF for 16 hours before being stimulated with either RANKL or M-CSF. To analyze protein expression during cell proliferation, BMMs were grown in $\alpha-10$ medium lacking M-CSF for 16-24 hours and then stimulated with $50 \mathrm{ng} / \mathrm{ml} \mathrm{M-CSF}$ for various times. Total cell lysates were collected and Western blot analysis was performed as described previously (52).

For immunoprecipitation, cells were washed in cold PBS and lysed on ice in buffer (20 mM Tris, pH 7.4, $150 \mathrm{mM} \mathrm{NaCl}, 0.2 \% \mathrm{NP}-40,1 \mathrm{mM}$ EDTA, and protease inhibitors). Lysates were passed through a 25 -gauge needle 10 times, incubated on ice for 30 minutes, and then clarified by centrifugation at $13,000 \mathrm{~g}$ for 15 minutes. One milligram of protein was incubated with 5-10 $\mu \mathrm{g}$ of antibodies overnight with rotation. Protein A/G agarose was then added and incubated with rotation for 3 hours at $4^{\circ} \mathrm{C}$. Immunoprecipitates were washed 4 times in lysis buffer, and solubilized proteins were separated by SDS-polyacrylamide gels.

Immunofluorescence and actin ring reformation assay. Osteoclasts cultured on bone slices were fixed with $4 \%$ paraformaldehyde in PBS for 20 minutes. Free aldehyde groups were quenched with $50 \mathrm{mM} \mathrm{NH}_{4} \mathrm{Cl}$ in PBS for 10 minutes, followed by washing and blocking in PBS $/ 0.2 \%$ BSA $/ 0.1 \%$ Saponin (PBSBS) for 30 minutes. Bone slices were incubated with primary and secondary antibodies in PBSBS for 45 minutes. F-actin was stained with Alexa Fluor 488-phalloidin. Samples were mounted with $90 \%$ glycerol/PBS and observed using a conventional microscope equipped with a charge-coupled device camera (Nikon USA) or a confocal laser scanning microscope (LSM Zeiss).

For actin ring re-formation assay, mature osteoclasts cultured on bone slices were washed twice with cold cytokine-free medium followed by incubation at $37^{\circ} \mathrm{C}$ for 30 minutes. RANKL $(100 \mathrm{ng} / \mathrm{ml})$ was then added for the indicated time. Bone slices were fixed with $4 \%$ paraformaldehyde in PBS for 20 minutes. F-actin and nuclei were stained with Alexa Fluor 488-phalloidin and Hoechst 33258, respectively. The number of total multinucleated osteoclasts and active osteoclasts with actin rings were counted under a conventional microscope equipped with a charge-coupled device camera (Nikon USA).

Cdc42 activation assay. Pre-osteoclasts stimulated with M-CSF or RANKL were lysed and Cdc42 activity was examined by an effector domain, GSTfusion pulldown protocol using EZ-detect Cdc42 activation kit (Pierce Biotechnology Inc.).

Proliferation and cell death ELISA assay. The BrdU ELISA was conducted using the cell proliferation Biotrak ELISA system (Amersham) as described previously (21). Cell death was analyzed in quadruplicate using cell death detection ELISAPLUS kit (Roche), which detects cytoplasmic histone-associated DNA fragmentation. All experiments were repeated two to three times.

Medium CTx-1 assay. Macrophages were cultured on bone with $100 \mathrm{ng} / \mathrm{ml}$ RANKL and 1/100 vol CMG14-12 supernatant for 5-6 days. Medium $(\alpha-10)$ was changed one day before harvesting. Medium CTx-1 concentration was determined using CrossLaps for Culture ELISA kit (Nordic Bioscience Diagnosis A/S).

Statistics. Data of 2-group comparisons were analyzed using a 2-tailed Student's $t$ test. Simultaneous comparison of more than 2 groups was performed using ANOVA. For all graphs and in the text, data are represented as mean $\pm \mathrm{SD}$.

\section{Acknowledgments}

This project was supported by grants AR032788, AR046523, AR054618, and AR057037 (to S.L. Teitelbaum), AR046852 (to F.P. Ross), AR055694 (to H. Zhao), and HL085362 (to Y. Zheng) from the NIH. The contents of this article are solely the responsibility of the authors and do not necessarily represent the official views of the NIH. We thank Shigeaki Kato for Ctsk $\mathrm{Cre}^{\mathrm{+}}$ mice, Patrick Brennwald for anti-LGL antibodies, Michael C. Ostrowski for anti-p-MITF antibody, and Paulette Shubert for assistance with manuscript preparation.

Received for publication April 23, 2009, and accepted in revised form February 24, 2010.

Address correspondence to: F. Patrick Ross or Haibo Zhao, Washington University School of Medicine, Department of Pathology and Immunology, Campus Box 8118, 660 South Euclid Avenue, 4940 Parkview Place, St. Louis, Missouri 63110, USA. Phone: 314.454.8463; Fax: 314.454.5505; E-mail: rossf@hss.edu (F.P. Ross); hzhao@uams.edu (H. Zhao). 
1. Zaidi M. Skeletal remodeling in health and disease. Nat Med. 2007;13(7):791-801.

2. Boyle WJ, Simonet WS, Lacey DL. Osteoclast differentiation and activation. Nature. 2003;423(6937):337-342.

3. Teitelbaum SL, Ross FP. Genetic regulation of osteoclast development and function. Nat Rev Genet. 2003;4(8):638-649.

4. Vaananen HK, Zhao H, Mulari M, Halleen JM. The cell biology of osteoclast function. J Cell Sci. 2000;113(Pt 3):377-381.

5. Jaffe AB, Hall A. Rho GTPases: biochemistry and biology. Annu Rev Cell Dev Biol. 2005;21:247-269.

6. Hall A. Rho GTPases and the control of cell behaviour. Biochem Soc Trans. 2005;33(Pt 5):891-895.

7. Chellaiah MA. Regulation of actin ring formation by rho GTPases in osteoclasts. J Biol Chem. 2005;280(38):32930-32943

8. Fukuda A, et al. Regulation of Osteoclast Apoptosis and Motility by Small GTPase Binding Protein Rac1. J Bone Miner Res. 2005;20(12):2245-2253.

9. Zhang D, et al. The small GTP-binding protein, rho $\mathrm{p} 21$, is involved in bone resorption by regulating cytoskeletal organization in osteoclasts. J Cell Sci. 1995;108(Pt 6):2285-2292.

10. Chellaiah MA, et al. Rho-A is critical for osteoclast podosome organization, motility, and bone resorption. J Biol Chem. 2000;275(16):11993-12002.

11. Destaing O, et al. A novel Rho-mDia2-HDAC6 pathway controls podosome patterning through microtubule acetylation in osteoclasts. J Cell Sci. 2005;118(Pt 13):2901-2911.

12. Faccio R, et al. Vav3 regulates osteoclast function and bone mass. Nat Med. 2005;11(3):284-290.

13. Calle $Y$, et al. WASp deficiency in mice results in failure to form osteoclast sealing zones and defects in bone resorption. Blood. 2004;103(9):3552-3561.

14. Hurst IR, Zuo J, Jiang J, Holliday LS. Actin-related protein $2 / 3$ complex is required for actin ring formation. J Bone Miner Res. 2004;19(3):499-506.

15. Kim H, et al. Selective inhibition of RANK blocks osteoclast maturation and function and prevents bone loss in mice. J Clin Invest. 2009;119(4):813-825.

16. Yang L, Wang L, Zheng Y. Gene targeting of Cdc42 and Cdc42GAP affirms the critical involvement of $\mathrm{Cdc} 42$ in filopodia induction, directed migration, and proliferation in primary mouse embryonic fibroblasts. Mol Biol Cell. 2006;17(11):4675-4685.

17. Nakamura T, et al. Estrogen prevents bone loss via estrogen receptor $\alpha$ and induction of Fas ligand in osteoclasts. Cell. 2007;130(5):811-823.

18. Wang L, Yang L, Burns K, Kuan CY, Zheng Y. Cdc42GAP regulates c-Jun $\mathrm{N}$-terminal kinase (JNK)mediated apoptosis and cell number during mammalian perinatal growth. Proc Natl Acad Sci U S A. 2005;102(38):13484-13489.

19. Bai S, et al. NOTCH1 regulates osteoclastogenesis directly in osteoclast precursors and indirectly via osteoblast lineage cells. J Biol Chem. 2008;283(10):6509-6518.

20. Barfod ET, et al. Cloning and expression of a human CDC42 GTPase-activating protein reveals a functional SH3-binding domain. J Biol Chem. 1993;268(35):26059-26062

21. Zhou P, Kitaura H, Teitelbaum SL, Krystal G, Ross FP, Takeshita S. SHIP1 negatively regulates proliferation of osteoclast precursors via Akt-dependent alterations in D-type cyclins and p27. J Immunol 2006;177(12):8777-8784.

22. Fesik SW. Insights into programmed cell death through structural biology. Cell. 2000; 103(2):273-282.

23. Akiyama T, et al. Regulation of osteoclast apoptosis by ubiquitylation of proapoptotic BH3-only Bcl-2 family member Bim. EMBO J. 2003;22(24):6653-6664.

24. Mansky KC, Sankar U, Han J, Ostrowski MC. Microphthalmia transcription factor is a target of the p38 MAPK pathway in response to receptor activator of NF-kappa B ligand signaling. J Biol Chem. 2002;277(13):11077-11083.

25. Tapon N, Hall A. Rho, Rac and Cdc42 GTPases regulate the organization of the actin cytoskeleton. Curr Opin Cell Biol. 1997;9(1):86-92.

26. Jurdic P, Saltel F, Chabadel A, Destaing O. Podosome and sealing zone: specificity of the osteoclast model. Eur J Cell Biol. 2006;85(3-4):195-202.

27. Kim HJ, et al. Glucocorticoids suppress bone formation via the osteoclast. J Clin Invest. 2006;116(8):2152-2160.

28. Lin D, Edwards AS, Fawcett JP, Mbamalu G, Scott JD, Pawson T. A mammalian PAR-3-PAR-6 complex implicated in Cdc42/Rac1 and aPKC signalling and cell polarity. Nat Cell Biol. 2000;2(8):540-547.

29. Joberty G, Petersen C, Gao L, Macara IG. The cellpolarity protein Par6 links Par3 and atypical protein kinase C to Cdc42. Nat Cell Biol. 2000;2(8):531-539.

30. Yamanaka T, et al. Mammalian Lgl forms a protein complex with PAR- 6 and aPKC independently of PAR-3 to regulate epithelial cell polarity. Curr Biol. 2003;13(9):734-743.

31. Plant PJ, et al. A polarity complex of mPar- 6 and atypical PKC binds, phosphorylates and regulates mammalian Lgl. Nat Cell Biol. 2003;5(4):301-308.

32. Suzuki A, Ohno S. The PAR-aPKC system: lessons in polarity. J Cell Sci. 2006;119(Pt 6):979-987.

33. Suzuki A, Akimoto K, Ohno S. Protein kinase C $\lambda / \iota(\mathrm{PKC} \lambda / \mathrm{\iota}):$ a PKC isotype essential for the development of multicellular organisms. J Biochem. 2003;133(1):9-16.

34. Hirai T, Chida K. Protein kinase C $\zeta$ (PKC $\zeta)$ : activation mechanisms and cellular functions. J Biochem. 2003;133(1):1-7.

35. Erickson JW, Cerione RA. Multiple roles for Cdc42 in cell regulation. Curr Opin Cell Biol. 2001;13(2):153-157.

36. Krum SA, et al. Estrogen protects bone by inducing Fas ligand in osteoblasts to regulate osteoclast survival. EMBO J. 2008;27(3):535-545.

37. Wu X, McKenna MA, Feng X, Nagy TR, McDonald JM. Osteoclast apoptosis: the role of Fas in vivo and in vitro. Endocrinology. 2003;144(12):5545-5555.

38. Zhao H, Ross FP, Teitelbaum SL. Unoccupied $\alpha v \beta 3$ integrin regulates osteoclast apoptosis by transmitting a positive death signal. Mol Endocrinol.
2005;19(3):771-780.

39. Gnesutta N, Qu J, Minden A. The serine/threonine kinase PAK4 prevents caspase activation and protects cells from apoptosis. J Biol Chem. 2001;276(17):14414-14419.

40. Bradley EW, Ruan MM, Oursler MJ. PAK1 is a novel MEK-independent raf target controlling expression of the IAP survivin in M-CSF-mediated osteoclast survival. J Cell Physiol. 2008;217(3):752-758.

41. Sharma SM, et al. MITF and PU.1 recruit p38 MAPK and NFATc1 to target genes during osteoclast differentiation. J Biol Chem. 2007;282(21):15921-15929.

42. Lee $\mathrm{ZH}, \mathrm{Kim} H \mathrm{HH}$. Signal transduction by receptor activator of nuclear factor kappa B in osteoclasts. Biochem Biophys Res Commun. 2003;305(2):211-214.

43. Ikeda F, et al. Critical roles of c-Jun signaling in regulation of NFAT family and RANKL-regulated osteoclast differentiation. J Clin Invest. 2004;114(4):475-484.

44. Saltel F, Chabadel A, Bonnelye E, Jurdic P. Actin cytoskeletal organisation in osteoclasts: a model to decipher transmigration and matrix degradation. Eur J Cell Biol. 2008;87(8-9):459-468.

45. Saltel F, Destaing O, Bard F, Eichert D, Jurdic P. Apatite-mediated actin dynamics in resorbing osteoclasts. Mol Biol Cell. 2004;15(12):5231-5241.

46. Miki H, Takenawa T. Regulation of actin dynamics by WASP family proteins. J Biochem. 2003;134(3):309-313.

47. Stradal TE, Scita G. Protein complexes regulating Arp2/3-mediated actin assembly. Curr Opin Cell Biol. 2006;18(1):4-10.

48. Millard TH, Sharp SJ, Machesky LM. Signalling to actin assembly via the WASP (Wiskott-Aldrich syndrome protein)-family proteins and the Arp $2 / 3$ complex. Biochem J. 2004;380(Pt 1):1-17.

49. Ory S, Brazier H, Blangy A. Identification of a bipartite focal adhesion localization signal in RhoU/Wrch-1, a Rho family GTPase that regulates cell adhesion and migration. Biol Cell. 2007;99(12):701-716.

50. Wang L, Yang L, Filippi MD, Williams DA, Zheng Y. Genetic deletion of Cdc42GAP reveals a role of Cdc42 in erythropoiesis and hematopoietic stem/ progenitor cell survival, adhesion, and engraftment. Blood. 2006;107(1):98-105.

51. Zhao H, Kitaura H, Sands MS, Ross FP, Teitelbaum SL, Novack DV. Critical role of beta3 integrin in experimental postmenopausal osteoporosis. JBone Miner Res. 2005;20(12):2116-2123.

52. Zhao H, Ito Y, Chappel J, Andrews NW, Teitelbaum SL, Ross FP. Synaptotagmin VII regulates bone remodeling by modulating osteoclast and osteoblast secretion. Dev Cell. 2008;14(6):914-925.

53. Takeshita S, Kaji K, Kudo A. Identification and characterization of the new osteoclast progenitor with macrophage phenotypes being able to differentiate into mature osteoclasts. J Bone Miner Res. 2000;15(8):1477-1488.

54. Takeshita $\mathrm{S}$, et al. c-Fms tyrosine 559 is a major mediator of M-CSF-induced proliferation of primary macrophages. J Biol Chem. 2007;282(26):18980-18990. 